\title{
The rational homology of spaces of long knots in codimension $>2$
}

\author{
PASCAL LAMBRECHTS \\ VICTOR TURCHIN \\ ISMAR VOLIĆ
}

\begin{abstract}
We determine the rational homology of the space of long knots in $\mathbb{R}^{d}$ for $d \geq 4$. Our main result is that the Vassiliev spectral sequence computing this rational homology collapses at the $E^{1}$ page. As a corollary we get that the homology of long knots (modulo immersions) is the Hochschild homology of the Poisson algebras operad with bracket of degree $d-1$, which can be obtained as the homology of an explicit graph complex and is in theory completely computable.

Our proof is a combination of a relative version of Kontsevich's formality of the little $d$-disks operad and of Sinha's cosimplicial model for the space of long knots arising from Goodwillie-Weiss embedding calculus. As another ingredient in our proof, we introduce a generalization of a construction that associates a cosimplicial object to a multiplicative operad. Along the way we also establish some results about the Bousfield-Kan spectral sequences of a truncated cosimplicial space.
\end{abstract}

57Q45; 57R40, 55P62

\section{Introduction}

A long knot is a smooth embedding $f: \mathbb{R} \hookrightarrow \mathbb{R}^{d}, d \geq 3$, that coincides with a fixed linear embedding $\epsilon: \mathbb{R} \hookrightarrow \mathbb{R}^{d}$ outside a compact set. We denote by $\operatorname{Emb}\left(\mathbb{R}, \mathbb{R}^{d}\right)$ the space of all long knots equipped with the weak $\mathcal{C}^{\infty}$ topology. This space, and in particular its homology, has been under investigation for more than twenty years. One of the first important tools in this study was a spectral sequence for computing the homology of spaces of knots constructed by Vassiliev in the late eighties [36]. This spectral sequence sparked a lot of interest, especially in dimension $d=3$, where it is related to the theory of finite type knot invariants.

Independently, Goodwillie and Weiss suggested another approach for the study of knots and more general spaces of embeddings which is now known as embedding calculus. In particular, it is suggested by Goodwillie, Klein and Weiss [15, Example 5.1.4] that this 
approach should also give a spectral sequence for computing the homology of spaces of long knots. Indeed, it later turned out that it does, and that this spectral sequence was equivalent to Vassiliev's. Goodwillie-Weiss embedding calculus for knots was developed further by Sinha in [31], who also emphasized the connection with the little $d$-disks operad in [30].

Vassiliev spectral sequence arises from a study of the discriminant set, ie the complement of the set of knots in the space of all smooth maps $\mathbb{R} \rightarrow \mathbb{R}^{d}$ with fixed behavior at infinity (or more precisely in some finite-dimensional approximation of that space). In other words, the discriminant set is the set of maps with singularities. This set admits a nice stratification, which in turn yields a natural filtration from which the spectral sequence is constructed. A classification of the singularities gives the $E^{1}$ page of this spectral sequence a combinatorial description in terms of certain graphs such as chord diagrams familiar from finite type knot theory. When $d \geq 4$, this spectral sequence converges to the homology of the space of long knots by Vassiliev [36, Section 6.6].

Vassiliev has conjectured a stable splitting of the resolved discriminant which would imply that his spectral sequence collapses at the $E^{1}$ page [38]. This collapse was proved rationally by Kontsevich in dimension $d=3$ along the diagonal $E_{-p, p}^{1}$. The proof uses the famous Kontsevich integral, a map that realizes all finite type invariants [16]. Kontsevich further claimed in [17, Theorem 2.3] that his integration approach can be generalized for $d \geq 4$ to give a proof of the collapse of the rational Vassiliev spectral sequence everywhere. In [7], Cattaneo, Cotta-Ramusino and Longoni filled in some details of that program and proved the collapse along the main diagonal. As far as we know, however, no complete proof of the rational collapse has yet appeared.

In this paper, we give a proof of Vassiliev's conjecture over the rationals.

Theorem 1.1 For $d \geq 4$, the Vassiliev spectral sequence computing the rational homology of the space of long knots $\operatorname{Emb}\left(\mathbb{R}, \mathbb{R}^{d}\right)$ collapses at the $E^{1}$ page.

To prove this theorem, we take a very different approach than that of Kontsevich, namely the Goodwillie-Weiss calculus of the embedding functor and Sinha's cosimplicial model for spaces of knots arising from this theory. Before explaining this further, it is convenient to introduce a variation of the space of long knots. Consider first the space of long immersions

$\operatorname{Imm}\left(\mathbb{R}, \mathbb{R}^{d}\right):=\left\{f: \mathbb{R} \rightarrow \mathbb{R}^{d} \mid\right.$

$f$ is an immersion that coincides with $\epsilon$ outside a compact set $\}$.

There is an inclusion $\iota: \operatorname{Emb}\left(\mathbb{R}, \mathbb{R}^{d}\right) \hookrightarrow \operatorname{Imm}\left(\mathbb{R}, \mathbb{R}^{d}\right)$. The homotopy fiber $\overline{\operatorname{Emb}}\left(\mathbb{R}, \mathbb{R}^{d}\right)$ of $\iota$ is called the space of long knots modulo immersions. By Smale-Hirsch theory [32], 
there is a weak equivalence $\operatorname{Imm}\left(\mathbb{R}, \mathbb{R}^{d}\right) \simeq \Omega S^{d-1}$. Moreover $\iota$ is null-homotopic by Sinha [30, Proposition 5.17], so we have a weak equivalence

$$
\overline{\mathrm{Emb}}\left(\mathbb{R}, \mathbb{R}^{d}\right) \simeq \operatorname{Emb}\left(\mathbb{R}, \mathbb{R}^{d}\right) \times \Omega^{2} S^{d-1}
$$

In [30] Sinha constructs a cosimplicial space

$$
\mathcal{K}_{d}^{\bullet}=\left(\mathcal{K}_{d}(0) \rightleftarrows \mathcal{K}_{d}(1) \stackrel{\rightleftarrows}{\rightleftarrows} \mathcal{K}_{d}(2) \cdots\right),
$$

where $\mathcal{K}_{d}=\left\{\mathcal{K}_{d}(n)\right\}_{n \geq 0}$ is a topological operad homotopy equivalent to the little $d-$ disks operad, called the Kontsevich operad. This operad turns out to be multiplicative, ie there exists a map $\{*\}_{n \geq 0} \rightarrow \mathcal{K}_{d}$ from the nonsymmetric associative topological operad which consists of the one-point space in each degree, to the Kontsevich operad. The cofaces and codegeneracies of $\mathcal{K}_{d}^{\bullet}$ are induced from this multiplicative structure via a general construction of Gerstenhaber and Voronov [12] which we recall in Section 2.2 using the McClure-Smith point of view [26, Section 3]. The main result of Sinha [30] is that for $d \geq 4$ the homotopy totalization of that cosimplicial space, $\operatorname{hoTot}\left(\mathcal{K}_{d}^{\bullet}\right)$, is weakly equivalent to $\overline{\operatorname{Emb}}\left(\mathbb{R}, \mathbb{R}^{d}\right)$.

The homology Bousfield-Kan spectral sequence [5] associated to $\mathcal{K}_{d}^{\bullet}$ converges to $\mathrm{H}_{*}\left(\operatorname{hoTot}\left(\mathcal{K}_{d}^{\bullet}\right)\right)$ when $d \geq 4[30$, Theorem 7.2]. Our main result is:

Theorem 1.2 For $d \geq 4$ the homology Bousfield-Kan spectral sequence associated to Sinha's cosimplicial space $\mathcal{K}_{d}^{\bullet}$ collapses at the $E^{2}$ page rationally.

Before looking at the consequences of this theorem, we give an overview of its proof. The key idea is a relative version of Kontsevich's theorem on the formality of the little $d$-disks operad [18, Section 3], which states that there is a chain of quasi-isomorphisms of operads between the singular chains of the little $d$-disks operad and its homology with real coefficients. This is therefore true for any operad weakly equivalent to the little $d$-disks operad. In particular there is a chain of quasi-isomorphisms $\mathrm{C}_{*}\left(\mathcal{K}_{d} ; \mathbb{R}\right) \simeq$ $\mathrm{H}_{*}\left(\mathcal{K}_{d} ; \mathbb{R}\right)$. If one could deduce from this the formality of the cosimplicial space $\mathcal{K}_{d}^{\bullet}$, the collapse of the homology Bousfield-Kan spectral sequence would immediately follow from Proposition 3.2. For this it would be enough to know, by McClure-Smith, that $\mathcal{K}_{d}$ is formal as a multiplicative operad. But this does not appear to be easy to establish (see Remark 7.4), and we do not know whether $\mathcal{K}_{d}^{\bullet}$ is formal.

This is why we make a detour through the Fulton-MacPherson operad $\mathcal{F}_{d}$. This operad is homotopy equivalent to $\mathcal{K}_{d}$ but is more suitable for proving formality results as those in [18]. It is not multiplicative, but it is "multiplicative up to homotopy" in the sense that it admits a map from the contractible $A_{\infty}$ Stasheff associahedral operad. The key formality statement that we prove is that when $d \geq 3, \mathcal{F}_{d}$ is formal as an operad which is "multiplicative up to homotopy" (Lemma 7.3). However, the problem is that we 
cannot directly associate to $\mathcal{F}_{d}$ a cosimplicial space in the spirit of McClure-Smith. We instead construct certain finite diagrams $\widehat{\mathcal{F}}_{d\{n\}}$ of spaces which we call fanic diagrams. These are built out of the up-to-homotopy multiplicative structure on $\mathcal{F}_{d}$ and are in a sense a rigid version of a "cosimplicial space up to homotopy" analogous to $\mathcal{K}_{d}^{\bullet}$, or more precisely analogous to its $n$-th truncation. Our formality statement implies that $\widehat{\mathcal{F}_{d\{n}}$ is formal (Theorem 7.1) and hence the homology Bousfield-Kan spectral sequence of the cosimplicial replacement of that diagram collapses at the $E^{2}$ page. Thus the same must be true for the cosimplicial replacement of the $n$-th truncation $\mathcal{K}_{d[n]}$ of $\mathcal{K}_{d}^{\bullet}$. Using the fact that, for each $n \geq 0$, the homology spectral sequence of $\mathcal{K}_{d[n]}$ collapses, along with a strong convergence result for Bousfield-Kan spectral sequences as it applies to $\mathcal{K}_{d}^{\bullet}$, we deduce Theorem 1.2.

We now come back to the Vassiliev spectral sequence and explain its link with the Bousfield-Kan spectral sequence. In his thesis, the second author found a more conceptual description of the $E^{1}$ page of the Vassiliev spectral sequence than the original combinatorial one arising from the classification of singularities in the discriminant set. To explain this, recall that the Poisson operad with bracket of degree $d-1$, $\mathcal{P O I S S} \mathcal{S}_{d-1}$, is the operad encoding Poisson algebras, ie graded commutative algebras equipped with a Lie bracket of degree $(d-1)$ which is a graded derivation with respect to the multiplication (see, for instance, Example (d) in Turchin [33, Section 1]). The work of Fred Cohen [8] implies that, for $d \geq 2, \mathcal{P O I S S}{ }_{d-1}$ is just the homology of the topological little $d$-disks operad. Since Poisson algebras admit an associative multiplication $m \in \mathcal{P O} \mathcal{O} S \mathcal{S}_{d-1}(2)$, we can define a differential

$$
\delta: \mathcal{P O I S S}{ }_{d-1}(n) \longrightarrow \mathcal{P O I S S}{ }_{d-1}(n+1)
$$

where $\delta=[m,-]$ is the Gerstenhaber bracket and $\delta^{2}=0$ by associativity [33, Section 3]. The homology of the cochain complex $\left(\mathcal{P O I S S} \mathcal{S}_{d-1}(*), \delta\right)$ is called the Hochschild homology of that multiplicative operad, denoted by $\mathrm{HH}_{*}\left(\mathcal{P O I S S} \mathcal{S}_{d-1}\right)$. This complex is in fact the deformation complex of the morphism of operads $\mathcal{A S S} \rightarrow$ $\mathcal{P O} \mathcal{I} \mathcal{S}_{d-1}$, where $\mathcal{A S S}$ is the associative algebras operad [19]. Finally, a slight variation of Vassiliev's spectral sequence for $\operatorname{Emb}\left(\mathbb{R}, \mathbb{R}^{d}\right)$ produces a spectral sequence for $\overline{\operatorname{Emb}}\left(\mathbb{R}, \mathbb{R}^{d}\right)$ and we have:

Theorem 1.3 (Turchin) The $E^{1}$ page of the Vassiliev spectral sequence computing $\mathrm{H}_{*}\left(\overline{\mathrm{Emb}}\left(\mathbb{R}, \mathbb{R}^{d}\right)\right)$ is isomorphic to the Hochschild homology of the Poisson operad with bracket of degree $d-1$.

This statement was first established for the Vassiliev spectral sequence for $\operatorname{Emb}\left(\mathbb{R}, \mathbb{R}^{d}\right)$ [33], and the above analogous result is a combination of [35, Theorem 8.4] and [34, Proposition 3.1 and Lemma 4.3]. 
On the other hand, the $E^{2}$ page of the Bousfield-Kan spectral sequence for $\mathcal{K}_{d}^{\bullet}$ is by definition the homology of the conormalization of the cosimplicial abelian group $\mathrm{H}_{*}\left(\mathcal{K}_{d}^{\bullet}\right)$. Since the cofaces are induced by the multiplicative structure on $\mathcal{K}_{d}$ and since the homology of that multiplicative topological operad is exactly the multiplicative Poisson operad, it is easy to deduce that this $E^{2}$ page is also isomorphic to $\mathrm{HH}_{*}\left(\mathcal{P O I S S _ { d - 1 }}\right)$ [30, Corollary 1.3]. This implies that the $E^{1}$ page of the Vassiliev spectral sequence for $\overline{\mathrm{Emb}}\left(\mathbb{R}, \mathbb{R}^{d}\right)$ and the $E^{2}$ page of the homology spectral sequence for $\mathcal{K}_{d}^{\bullet}$ are isomorphic. It can be shown that this isomorphism is just a regrading [35, Proposition 0.1]. Since both of these spectral sequences converge to $\mathrm{H}_{*}\left(\overline{\mathrm{Emb}}\left(\mathbb{R}, \mathbb{R}^{d}\right) ; \mathbb{Q}\right)$ for $d \geq 4$, Theorem 1.2 implies that the Vassiliev spectral sequence collapses at the $E^{1}$ page. As an immediate consequence of this and (1-1) we have the following:

Corollary 1.4 For $d \geq 4$,

$$
\begin{aligned}
\mathrm{H}_{*}\left(\operatorname{Emb}\left(\mathbb{R}, \mathbb{R}^{d}\right) ; \mathbb{Q}\right) \otimes \mathrm{H}_{*}\left(\Omega^{2} S^{d-1} ; \mathbb{Q}\right) & \cong \mathrm{H}_{*}\left(\overline{\operatorname{Emb}}\left(\mathbb{R}, \mathbb{R}^{d}\right) ; \mathbb{Q}\right) \\
& \cong \mathrm{HH}_{*}\left(\mathcal{P O I S S} \mathcal{S}_{d-1}\right)
\end{aligned}
$$

The rational homology of $\Omega^{2} S^{d-1}$ is isomorphic to a free graded commutative algebra on one or two generators depending on the parity of $d$, so it is very simple. In addition, the $E^{2}$ page of the Bousfield-Kan spectral sequence for $\mathcal{K}_{d}^{\bullet}$, or equivalently the Hochschild homology of the Poisson operad, can be expressed as the homology of an explicit cochain complex described in terms of the homology of configuration spaces in $\mathbb{R}^{d}$ which are well understood. In particular, there is an algorithm for computing this homology which has been used by the second author [35]. Thus, in theory, $\mathrm{H}_{*}\left(\operatorname{Emb}\left(\mathbb{R}, \mathbb{R}^{d}\right) ; \mathbb{Q}\right)$ is completely computable. However, it appears that the algorithmic complexity is exponential and the only feasible computations are all still in low degrees. What is necessary, and is still lacking, is a deeper understanding of the structure of $\mathrm{HH}_{*}\left(\mathcal{P O I S S} \mathcal{S}_{d-1}\right)$.

It is easy to see that the homology of the cosimplicial space $\mathcal{K}_{d}^{\bullet}$ depends, up to an obvious regrading, only on the parity of $d$. This implies that, up to regrading, all the $E^{2}$ pages of the homology Bousfield-Kan spectral sequences of $\mathcal{K}_{d}^{\bullet}$ for $d$ even (respectively $d$ odd) are isomorphic. This completes the proof of the characterization of the rational homology of spaces of long knots as stated by Kontsevich [17, Theorem 2.3]. Furthermore, the operation of stacking of long knots gives a multiplication which makes $\operatorname{Emb}\left(\mathbb{R}, \mathbb{R}^{d}\right)$ into an $H$-space. Therefore the rational homotopy type of $\operatorname{Emb}\left(\mathbb{R}, \mathbb{R}^{d}\right)$ for $d \geq 4$ is completely determined by its rational homology and is thus virtually known by our main theorem.

Coming back to the original Vassiliev conjecture, notice that by Turchin [34, Theorem 5.1] and results of Vassiliev [37, Section IV], the $E^{1}$ page of the Vassiliev spectral 
sequence for $\operatorname{Emb}\left(\mathbb{R}, \mathbb{R}^{d}\right)$ and the corresponding $\overline{E^{1}}$ page of the Vassiliev spectral sequence for $\overline{\mathrm{Emb}}\left(\mathbb{R}, \mathbb{R}^{d}\right)$ are related by an isomorphism $E^{1} \otimes \mathrm{H}_{*}\left(\Omega^{2} S^{d-1}\right) \cong \overline{E^{1}}$. Therefore Corollary 1.4 and the isomorphism $\overline{E^{1}} \cong \mathrm{HH}_{*}\left(\mathcal{P O} \mathcal{I} \mathcal{S}_{d-1}\right)$ from Theorem 1.3 imply the collapse at $E^{1}$ of the classical Vassiliev spectral sequence for $\operatorname{Emb}\left(\mathbb{R}, \mathbb{R}^{d}\right)$, which is our promised Theorem 1.1.

The homotopy Bousfield-Kan spectral sequence computing the homotopy groups of $\operatorname{Emb}\left(\mathbb{R}, \mathbb{R}^{d}\right)$ from the cosimplicial abelian group $\pi_{*}\left(\mathcal{K}_{d}^{\bullet}\right)$ has been studied elsewhere by Arone et al [1]. Using the remarkable fact that the little $d$-disks operad is not only formal (ie rationally determined by its homology), but also coformal (ie rationally determined by its homotopy), it has been shown in [1] that Theorem 1.2 implies that the homotopy spectral sequence tensored with the rationals also collapses at the $E^{2}$ page. A strong connection between the $E^{2}$ pages of the rational homology and the homotopy spectral sequences is exhibited by the first two authors [20] where it is also shown that the homotopy groups of the space of long knots are also the homology of a certain graph complex, smaller than the one used for computing the homology of $\operatorname{Emb}\left(\mathbb{R}, \mathbb{R}^{d}\right)$. Some computations in this $E^{2}$ page appeared in Scannell and Sinha [28].

A natural next question is whether our approach gives the collapse of the Vassiliev spectral sequence for long knots in $\mathbb{R}^{3}$. There are many difficult issues here, not the least of which is that it is not even clear what the Vassiliev spectral sequence converges to. However, we are nevertheless able to prove a certain collapsing result. Indeed, Proposition 8.1 states that the homology Bousfield-Kan spectral sequence of the cosimplicial replacement of the $n$-th truncation $\mathcal{K}_{d[n]}$ collapses at $E^{2}$. This is true for $d \geq 3$, but to deduce the collapse of the Bousfield-Kan spectral sequence for $\mathcal{K}_{d}^{\bullet}$ itself we need some convergence hypotheses which are only true for $d \geq 4$. It at least seems plausible that this spectral sequence collapses for $d=3$. Another interesting question is whether our result can be extended over the integers.

Finally, it is possible to extend our results to spaces of embeddings of more general manifolds than $\mathbb{R}$ in $\mathbb{R}^{d}$. This is done by generalizing the following slogan which summarizes our proof of Theorem 1.2:

Goodwillie-Weiss embedding calculus $+$

Kontsevich formality of the little $d$-disks operad

collapse of spectral sequences for spaces of embeddings

This slogan was taken much further by Arone, the first author and Volić [2]. The authors of that paper use Weiss' orthogonal calculus to prove a collapse result for a spectral sequence computing the rational homology of $\overline{\operatorname{Emb}}\left(M, \mathbb{R}^{d}\right)$ for any compact manifold $M$ and $d$ large enough. In particular, it is shown that for large $d$, the rational 
homology of the space of embeddings modulo immersions, $\mathrm{H}_{*}\left(\overline{\operatorname{Emb}}\left(M, \mathbb{R}^{d}\right) ; \mathbb{Q}\right)$, depends only on the rational homotopy type of $M$.

\subsection{Organization of the paper}

In Section 2 we review basic facts on cosimplicial spaces and diagrams. We also recall Sinha's cosimplicial model for the space of long knots, the notion of a multiplicative operad and the associated Gerstenhaber-Voronov/McClure-Smith cosimplicial object and the definitions of the Kontsevich and Fulton-MacPherson operads. Formality, which comes from rational homotopy theory, is recalled in Section 3 where we also prove that the homology Bousfield-Kan spectral sequence of a formal cosimplicial space, or more generally of the cosimplicial replacement of a formal finite diagram, collapses at the $E^{2}$ page. In Section 4 we study a condition on a cosimplicial space that guarantees that the Bousfield-Kan spectral sequences for its truncations converge. In Section 5 we introduce the category of fans, which is a variation of the truncated cosimplicial category $\Delta[n]$, and in Section 6 show how to associate to any morphism of nonsymmetric operads a diagram shaped on the category of fans. This generalizes the Gerstenhaber-Voronov/McClureSmith cosimplicial diagram associated to a multiplicative operad. In Section 7 we prove a relative version of Kontsevich's formality of the little $d$-disks operad and deduce the formality of the fanic diagrams associated to the Kontsevich multiplicative operad. In Section 8 , we collect these results to give a proof of our main theorem, Theorem 1.2.

Acknowledgments We are very grateful to Greg Arone for many conversations and encouragement, as well as for arranging several visits of the first author to University of Virginia. We also thank Peter Bousfield, Emmanuel Dror-Farjoun and Bill Dwyer for answering our many questions on the Bousfield-Kan spectral sequences, Tom Goodwillie and Dev Sinha for fruitful conversations, and Ryan Budney for help with figures.

The first author is Chercheur Qualifié at FNRS. The second author was supported in part by grants NSH-1972.2003.01 and RFBR 05-01-01012a. The third author was supported in part by the National Science Foundation grant DMS 0504390.

\section{The Kontsevich and Fulton-MacPherson operads and Sinha's cosimplicial model for the space of long knots}

In this section we review Sinha's cosimplicial model for the space of long knots and its relation to the Kontsevich and Fulton-MacPherson operads. To start, we review some standard facts about cosimplicial spaces and diagrams as well as the construction of a cosimplicial space associated to a multiplicative operad. 


\subsection{Basic terminology and facts: Cosimplicial spaces, homotopy totaliza- tions, Bousfield-Kan spectral sequences, cosimplicial replacement of diagrams and left cofinal functors}

The standard references for the following basic terminology and facts about cosimplicial objects are Bousfield and Kan [6, X] and Weibel [39, Chapter 8].

The simplicial category $\Delta$ has ordered sets $[n]:=\{0,1,2, \ldots, n\}, n \geq 0$, as objects and order-preserving maps as morphisms. All morphisms in $\Delta$ are compositions of cofaces $d^{i}:[n] \rightarrow[n+1]$ and codegeneracies $s^{j}:[n] \rightarrow[n-1]$. A cosimplicial object in a category $\mathcal{C}$ is a covariant functor from $\Delta$ to $\mathcal{C}$. Dually, a simplicial object in $\mathcal{C}$ is a contravariant functor from $\Delta$ to $\mathcal{C}$. In particular a (pointed) cosimplicial space is a covariant functor

$$
X^{\bullet}: \Delta \longrightarrow \text { Top } \quad\left(\text { or } \mathrm{Top}_{*}\right)
$$

from the simplicial category to the category of (pointed) spaces. The standard cosimplicial space is the cosimplicial space $\Delta^{\bullet}$ where $\Delta^{n}$ is the standard geometric $n-$ simplex, the cofaces are defined from the inclusions of faces $\Delta^{n} \hookrightarrow \Delta^{n+1}$, and the codegeneracies are suitable affine projections $\Delta^{n} \rightarrow \Delta^{n-1}$.

The totalization of a cosimplicial space $X^{\bullet}$, denoted by Tot $X^{\bullet}$, is the space of natural maps from the standard cosimplicial space $\Delta^{\bullet}$ to $X^{\bullet}$,

$$
\operatorname{Tot} X^{\bullet}:=\operatorname{Hom}_{\Delta}\left(\Delta^{\bullet}, X^{\bullet}\right) \text {. }
$$

When the cosimplicial space $X^{\bullet}$ is fibrant [6, X.4.6], $\operatorname{Tot}\left(X^{\bullet}\right)$ is homotopy equivalent to the homotopy $\operatorname{limit}_{\text {holim }} X^{\bullet}$ [6, XI.4.4]. As explained in [5, Section 2.7], the techniques of [6, pages 279-280] imply that any cosimplicial space $X^{\bullet}$ admits a weakly equivalent fibrant functorial replacement $\widetilde{X}^{\bullet}$. One then defines the homotopy totalization of $X^{\bullet}$ by

$$
\text { hoTot } X^{\bullet}:=\operatorname{Tot} \tilde{X}^{\bullet} \text {. }
$$

Since by definition $\tilde{X}^{\bullet}$ is fibrant and weakly equivalent to $X^{\bullet}$, hoTot $X^{\bullet}$ is always weakly equivalent to holim $X^{\bullet}$. This homotopy totalization hoTot is also weakly equivalent to the homotopy invariant totalization Tot used in [30].

Consider the full subcategory $\Delta[n] \subset \Delta$ consisting of objects $[0], \ldots,[n]$. The $n-t h$ truncation of a cosimplicial object $X^{\bullet}$ in $\mathcal{C}$ is the composite

$$
X_{[n]}: \Delta[n] \hookrightarrow \Delta \stackrel{X^{\bullet}}{\longrightarrow} \mathcal{C} .
$$

The $n$-th partial homotopy totalization of $X^{\bullet}$ is defined as

$$
\operatorname{hoTot}^{n}\left(X^{\bullet}\right):=\underset{\Delta[n]}{\operatorname{holim}} X_{[n]} .
$$


To any cosimplicial space $X^{\bullet}$ one can associate a second quadrant homology BousfieldKan spectral sequence with coefficients in an abelian group $A$ [5], and a homotopy Bousfield-Kan spectral sequence if $X^{\bullet}$ is pointed [6]. These converge under favorable circumstances to $\mathrm{H}_{*}\left(\right.$ hoTot $\left.X^{\bullet} ; A\right)$ or $\pi_{*}\left(\right.$ hoTot $\left.X^{\bullet}\right)$. We state certain strong convergence conditions in Section 4.

A diagram of spaces is a covariant functor $F: I \rightarrow$ Top where $I$ is a small category called the shape of the diagram. We will also use the notion of a cosimplicial replacement of the diagram $F$ as defined in [6, XI.5], denoted by $\Pi^{\bullet} F$. The $n$-th term of this cosimplicial space is given by

$$
\Pi^{n} F:=\prod_{u=\left(i_{0} \leftarrow \ldots \stackrel{f_{1}}{\leftarrow} i_{n}\right) \in \mathrm{N}_{n}(I)} F\left(i_{0}\right)
$$

where the simplicial set $\mathrm{N}_{\bullet}(I)$ is the nerve of the category $I$. The cofaces and codegeneracies are obtained as suitable diagonal maps and projections. By [6, XI.5.2], the (homotopy) totalization of this cosimplicial space is weakly equivalent to the homotopy limit of $F$, ie

$$
\operatorname{hoTot}\left(\Pi^{\bullet} F\right) \simeq \underset{I}{\operatorname{holim}} F .
$$

If $\theta: J \rightarrow I$ is a functor then the $I$-diagram $F$ induces a $J$-diagram $\theta^{*} F:=F \circ \theta$. This change of shapes functor $\theta$ is said to be left cofinal if for every object $i$ in $I$, the overcategory $\theta \downarrow i$ is contractible (see [6, XI.9] with $\theta \downarrow i$ defined and denoted by $\theta / i$ in $[6, \mathrm{XI} .2 .2])$. In this case there is a weak equivalence

$$
\underset{I}{\operatorname{holim}} F \simeq \underset{J}{\operatorname{holim}} \theta^{*} F .
$$

\subsection{The construction of a cosimplicial object associated to a multiplicative operad following McClure and Smith}

Here we recall the notion of the cosimplicial object associated to a multiplicative operad from [26, Section 3].

Let $(\mathcal{C}, \otimes, \mathbf{1})$ be a symmetric monoidal category where the object $\mathbf{1}$ is the unit for $\otimes$. A nonsymmetric operad $\mathcal{O}=\{\mathcal{O}(n)\}_{n \geq 0}$ is a collection of objects of $\mathcal{C}$ with all the properties of an operad except those having to do with the actions of the symmetric groups [25, Definition 3.12]. Notice that as part of the definition there is a unit morphism id: $\mathbf{1} \rightarrow \mathcal{O}(1)$ and we also suppose that a (nonsymmetric) operad has an object in degree 0 , contrary to the definition in [24]. We denote by

$$
\circ_{i}: \mathcal{O}(p) \otimes \mathcal{O}(q) \longrightarrow \mathcal{O}(p+q-1), \quad 1 \leq i \leq p,
$$


the usual insertion operations induced by the operad structure as defined for example by Markl, Shnider and Stasheff [24, page 7]. The associative nonsymmetric operad $\mathcal{A S S}$ is defined by $\mathcal{A S S}(n)=\mathbf{1}$ for each $n \geq 0$, with operadic structure maps the standard isomorphisms $\mathbf{1}^{\otimes(1+n)} \cong \mathbf{1}$.

A multiplicative operad is a nonsymmetric operad $\mathcal{O}$ equipped with a morphism of nonsymmetric operads $\mu: \mathcal{A S S} \rightarrow \mathcal{O}$ [12; 26, Definition 3.1 and Remark 3.2 (i)]. Such a multiplicative structure on $\mathcal{O}$ is equivalent to having morphisms $e: \mathbf{1} \rightarrow \mathcal{O}(0)$ and $m: \mathbf{1} \rightarrow \mathcal{O}(2)$ satisfying

$$
m \circ_{1} m=m \circ_{2} m \text { and } m \circ_{1} e=m \circ_{2} e=\mathrm{id} .
$$

One can associate a cosimplicial object $\mathcal{O}^{\bullet}$ to any multiplicative operad $\mathcal{O}$ [26, Section 3] by defining $\mathcal{O}^{n}=\mathcal{O}(n)$ with the cofaces and codegeneracies given by the following formulas. For $x \in \mathcal{O}(n)$,

$$
\begin{aligned}
d^{0}(x) & =m \circ_{2} x, \\
d^{i}(x) & =x \circ_{i} m, \quad \text { for } 1 \leq i \leq n, \\
d^{n+1}(x) & =m \circ_{1} x, \\
s^{j}(x) & =x \circ_{j} e, \quad \text { for } 1 \leq j+1 \leq n .
\end{aligned}
$$

It is easy to check that the cosimplicial identities are consequences of Equation (2-1). There are also obvious dual notions of a cooperad, of a coassociative cooperad (also denoted by $\mathcal{A S S}$ ), of a comultiplicative cooperad which has a map too the coassociative operad, and of a simplicial object associated to a comultiplicative cooperad.

\subsection{The Kontsevich multiplicative operad and Sinha's cosimplicial model}

Fix $d \geq 1$ and recall that a linear embedding $\epsilon: \mathbb{R} \hookrightarrow \mathbb{R}^{d}$ has also been fixed.

The Kontsevich operad $\mathcal{K}_{d}=\left\{\mathcal{K}_{d}(n)\right\}_{n \geq 0}$ is defined and studied in [30, Definition 4.1 and Theorem 4.5]. Each space $\mathcal{K}_{d}(n)$ is obtained as a suitable compactification of the ordered configuration space of $n$ points in $\mathbb{R}^{d}$ modulo the action of $\mathbb{R} \ltimes \mathbb{R}^{d}$ by scaling and translation. Another feature is that colinear configurations are identified. More precisely, $\mathcal{K}_{d}(n)$ is the closure of the image of the map

$$
\alpha_{*}=\left(\alpha_{i j}\right)_{1 \leq i<j \leq n}: C\left(n, \mathbb{R}^{d}\right) \longrightarrow \prod_{1 \leq i<j \leq n} S^{d-1}
$$

where $C\left(n, \mathbb{R}^{d}\right)$ is the space of configurations of $n$ points in $\mathbb{R}^{d}$ and $\alpha_{i j}: C\left(n, \mathbb{R}^{d}\right) \rightarrow$ $S^{d-1}$ is defined by $\alpha_{i j}\left(x_{1}, \ldots, x_{n}\right)=\left(x_{i}-x_{j}\right) /\left\|x_{i}-x_{j}\right\|$. It can be shown that this operad is homotopy equivalent to the classical little $d$-disks operad. 
The spaces $\mathcal{K}_{1}(n)$ turn out to be homeomorphic to the discrete symmetric group $\Sigma_{n}$ on $n$ letters, since all configuration on $\mathbb{R}$ are colinear. Let $\mathcal{K}_{1}^{(0)}(n)$ be the path-connected component of $\mathcal{K}_{1}(n)$ corresponding to the linearly ordered configuration $(1, \ldots, n)$ on the line. Since $\mathcal{K}_{1}^{(0)}(n)$ is a one-point space, it is clear that $\mathcal{K}_{1}^{(0)}=\mathcal{A S S}$, the associative nonsymmetric operad in the monoidal cartesian category of spaces.

The linear embedding $\epsilon$ induces a morphism of operads

$$
\epsilon_{\#}: \mathcal{K}_{1} \longrightarrow \mathcal{K}_{d}
$$

which sends a configuration on the line to its image under $\epsilon$ in $\mathbb{R}^{d}$. This restricts to a morphism

$$
\epsilon_{\#}: \mathcal{K}_{1}^{(0)}=\mathcal{A S S} \longrightarrow \mathcal{K}_{d}
$$

which endows $\mathcal{K}_{d}$ with the structure of a multiplicative operad.

One can thus associate a cosimplicial space $\mathcal{K}_{d}^{\bullet}$, which we will call Sinha's cosimplicial space, to $\mathcal{K}_{d}$, as outlined in Section 2.2. In more detail, this is a cosimplicial space

$$
\mathcal{K}_{d}^{\bullet}=\left(\mathcal{K}_{d}(0) \rightleftarrows \mathcal{K}_{d}(1) \stackrel{\rightleftarrows}{\rightleftarrows} \mathcal{K}_{d}(2) \cdots\right),
$$

where $\mathcal{K}_{d}(n)$ has the homotopy type of the space of configurations of $n$ points in $\mathbb{R}^{d}$. Cofaces $d^{i}$ corresponds to "doubling" the $i$-th point of the configuration "infinitesimally" in the direction given by $\epsilon$ and codegeneracies $s^{i}$ forget the $i$-th point in the configuration. This is explained in detail in $[31 ; 30]$.

We have the following important result due to Sinha.

Theorem 2.1 [30, Corollary 1.2] For $d \geq 4$, the space of long knots modulo immersion is weakly equivalent to the homotopy totalization of the cosimplicial space associated to the Kontsevich operad, ie

$$
\overline{\operatorname{Emb}}\left(\mathbb{R}, \mathbb{R}^{d}\right) \simeq \operatorname{hoTot}\left(\mathcal{K}_{d}^{\bullet}\right) .
$$

\subsection{The Fulton-MacPherson operad}

We recall here the Fulton-MacPherson operad and its relation to the Kontsevich operad. We will need this operad to establish certain formality results. Our main reference is Sinha [29], although this operad is also studied in Axelrod and Singer [3] Gaiffi [11], Getzler and Jones [13], Kontsevich [18] and Markl [23].

The Fulton-MacPherson operad, $\mathcal{F}_{d}=\left\{\mathcal{F}_{d}(n)\right\}_{n \geq 0}$, is a topological operad whose $n$-th term is also a compactification of $C\left(n, \mathbb{R}^{d}\right)$ modulo scaling and translation. The difference between this and the compactification defining the Kontsevich operad is that 
no identification of the collinear configurations takes place. More precisely, $\mathcal{F}_{d}(n)$ is defined as the closure of the image of the map

$$
\left(\alpha_{*}, \beta_{*}\right): C\left(n, \mathbb{R}^{d}\right) \rightarrow \prod_{1 \leq i<j \leq n} S^{d-1} \times \prod_{1 \leq i<j<k \leq n}[0, \infty]
$$

where $\alpha_{*}=\left(\alpha_{i j}\right)_{1 \leq i<j \leq n}$ is as in (2-2) and $\beta_{*}=\left(\beta_{i j k}\right)_{1 \leq i<j<k \leq n}$ is defined by $\beta_{i j k}\left(x_{1}, \ldots, x_{n}\right)=\left\|x_{i}-x_{j}\right\| /\left\|x_{i}-x_{k}\right\|[29$, Definition 4.11]. There is a morphism of operads

$$
q: \mathcal{F}_{d} \longrightarrow \mathcal{K}_{d}
$$

induced by the obvious projection between the target spaces of maps (2-3) and (2-2). Each $q: \mathcal{F}_{d}(n) \rightarrow \mathcal{K}_{d}(n)$ is a homotopy equivalence [29, Corollary 5.9] (see also [30, Theorem 4.2]), as is the map of operads (2-4) [27] (see also [22, Section 2]).

Denote by $\mathcal{F}_{1}^{(0)}(n)$ the path component in $\mathcal{F}_{1}(n)$ containing the linearly ordered configuration $(1, \ldots, n)$ on the line. This defines a nonsymmetric operad $\mathcal{F}_{1}^{(0)}$ which is homeomorphic to the Stasheff operad. In particular $\mathcal{F}_{1}^{(0)}(n)$ is the $n$-th associahedron which is a convex polytope of dimension $n-2$ (or 0 for $n<2$ ) [29, Section 4.4]. The linear embedding $\epsilon$ induces a morphism $\epsilon_{\#}: \mathcal{F}_{1}^{(0)} \rightarrow \mathcal{F}_{d}$ and we have a commutative diagram of nonsymmetric operads

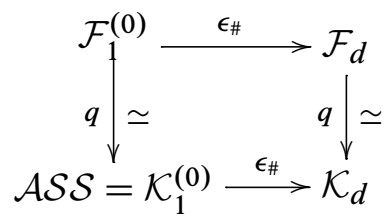

where the vertical maps are homotopy equivalences.

The operad $\mathcal{F}_{d}$ is not multiplicative but since each $\mathcal{F}_{1}^{(0)}(n)$ is contractible, $\epsilon_{\#}$ in a sense endows $\mathcal{F}_{d}$ with a multiplicative structure "up to homotopy". Thus we cannot apply the Gerstenhaber-Voronov/McClure-Smith construction directly to obtain a cosimplicial space out of $\mathcal{F}_{d}$, but we will see in Section 6 that one can associate a more general diagram to $\mathcal{F}_{d}$ which generalizes this construction.

\section{Formality and collapse of the homology Bousfield-Kan spectral sequence}

In this section we recall the notion of a formal diagram in rational homotopy theory and we show that the homology Bousfield-Kan spectral sequence of a formal cosimplicial 
space collapses at the $E^{2}$ page. We also show that formality of a finite diagram is preserved by passing to its cosimplicial replacement.

We first review some classical notions in rational homotopy theory for which Félix, Halperin and Thomas [10] is the standard reference. Let $\mathbb{K}$ be a field of characteristic 0 and denote by CDGA the category of commutative differential graded algebras. Let

$$
A_{\mathrm{PL}}(-; \mathbb{K}): \text { Top } \rightarrow \text { CDGA }
$$

be Sullivan's functor of piecewise polynomial forms as defined in [10, Section 10 (c)] and recall that, for a space $X, H\left(A_{\mathrm{PL}}(X ; \mathbb{K})\right) \cong H^{*}(X ; \mathbb{K})$. In fact $A_{\mathrm{PL}}(-; \mathbb{K})$ is naturally connected by a zigzag of quasi-isomorphisms of cochain complexes to the singular cochains $C^{*}(-; \mathbb{K})[10$, Section $10(\mathrm{e})]$. The definition of a formal space was introduced in [9], and it can be generalized to diagrams as follows.

Definition 3.1 Let $I$ be a small category and let $\mathbb{K}$ be a field of characteristic 0 . A functor $A: I \rightarrow$ CDGA is called formal if it is connected by a zigzag of natural quasi-isomorphism to its homology $\mathrm{H}(A)$. A diagram of spaces $F: I \rightarrow$ Top is called $\mathbb{K}$-formal if the contravariant functor $A_{\mathrm{PL}}(F ; \mathbb{K}): I \rightarrow \mathrm{CDGA}$ is formal.

As a special case, a cosimplicial space $X^{\bullet}$ is formal if the diagram $X^{\bullet}: \Delta \rightarrow$ Top is formal. Our main interest in this is the following collapsing result.

Proposition 3.2 Let $X^{\bullet}$ be a cosimplicial space and let $\mathbb{K}$ be a field of characteristic 0 . If the cosimplicial space $X^{\bullet}$ is $\mathbb{K}$-formal then the homology Bousfield-Kan spectral sequence for $X^{\bullet}$ with coefficients in any field of characteristic 0 collapses at the $E^{2}$ page.

Proof We consider the homology Bousfield-Kan spectral sequence as constructed in [5, Section 2.1]. For a cosimplicial chain complex $V_{*}^{\bullet}$, denote by $\left\{E^{r}\left(V_{*}^{\bullet}\right)\right\}_{r \geq 0}$ the spectral sequence induced by the filtration by cosimplicial degree in the associated total complex of the bicomplex $\prod_{m \geq 0} N^{m}\left(V_{*}^{\bullet}\right)$. Here $N^{*}$ is the conormalization as defined in [5, Section 2] or in [39, Chapter 8]. It is clear that $\left\{E^{r}\left(C_{*}\left(X^{\bullet} ; \mathbb{K}\right)\right)\right\}_{r \geq 0}$ coincides from the $E^{2}$ page with the homology Bousfield-Kan spectral sequence of $X^{\bullet}$. The $\mathbb{K}$-formality of the cosimplicial space and the natural equivalence between $A_{\mathrm{PL}}$ and singular cochains imply that there is a zigzag of quasi-isomorphisms

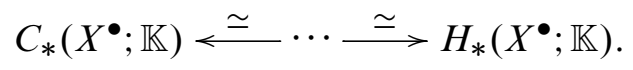

Therefore the spectral sequence $\left\{E^{r}\left(C_{*}\left(X^{\bullet} ; \mathbb{K}\right)\right)\right\}_{r \geq 0}$ coincides from the $E^{1}$ page with $\left\{E^{r}\left(H_{*}\left(X^{\bullet} ; \mathbb{K}\right)\right)\right\}_{r \geq 0}$. But the latter spectral sequence collapses at $E^{2}$ because each $H_{*}\left(X^{n} ; \mathbb{K}\right)$ is a chain complex with 0 differential so that the vertical differential 
in the associated bicomplex is trivial. This proves the statement for $\mathbb{K}$. We also have an isomorphism of spectral sequences

$$
\left\{E^{r}\left(C_{*}\left(X^{\bullet} ; \mathbb{K}\right)\right)\right\}_{r \geq 0} \cong\left\{E^{r}\left(C_{*}\left(X^{\bullet} ; \mathbb{Q}\right)\right) \otimes_{\mathbb{Q}} \mathbb{K}\right\}_{r \geq 0},
$$

and therefore the spectral sequence with rational coefficients collapses at the same page. Applying this argument in the opposite direction proves the result for any field of characteristic 0 .

If we could prove that Sinha's cosimplicial space $\mathcal{K}_{d} \bullet$ was formal we would deduce immediately from the last proposition the collapse of the associated homology BousfieldKan spectral sequence as claimed in Theorem 1.2. Unfortunately, it seems difficult to prove formality of $\mathcal{K}_{d} \bullet$ directly, so we will prove formality of some other diagrams approximating $\mathcal{K}_{d} \bullet$ instead. But in order to deduce collapsing results from formality of these auxiliary diagrams, we now need to show that the cosimplicial replacement of a formal finite diagram is a formal cosimplicial space.

A category $I$ is said to be finite if it has a finite number of morphisms (hence of objects).

Proposition 3.3 Let $I$ be a finite category, let $\mathbb{K}$ be a field of characteristic 0 , and let $F: I \rightarrow$ Top be a diagram. If $F$ is $\mathbb{K}$-formal then so is its cosimplicial replacement $\Pi^{\bullet} F$.

Proof Recall from [6, XI.5] and from the end of Section 2.1 the definition of the cosimplicial replacement $\Pi^{\bullet} F$. Inspired by this construction we associate to a contravariant functor $A: I \rightarrow$ CDGA a simplicial CDGA, which we denote by $\otimes_{\bullet} A$ and define as follows. Let $\mathrm{N}_{\bullet}(I)$ be the nerve of $I$ and denote by

$$
u=\left(i_{0} \stackrel{f_{1}}{\leftarrow} \ldots \stackrel{f_{n}}{\leftarrow} i_{n}\right)
$$

a typical element of $\mathrm{N}_{n}(I)$. The $n-$ th term of $\otimes_{\bullet} A$ is given by

$$
(\otimes \bullet A)_{n}:=\bigotimes_{u \in \mathrm{N}_{n}(I)} A\left(i_{0}\right)
$$

Before defining the cofaces, notice that for $u \in \mathrm{N}_{n}(I)$, taking all possible factorizations $f_{i}=f^{\prime \prime} \circ f^{\prime}$ for a fixed $1 \leq i \leq n$, postcomposing by all possible maps $g: i_{-1} \leftarrow i_{0}$ with source $i_{0}$, or precomposing with all possible maps $h: i_{n} \leftarrow i_{n+1}$ with target $i_{n}$ 
gives isomorphisms

$$
\left(\otimes_{\bullet} A\right)_{n+1} \cong \begin{cases}\bigotimes_{u \in \mathrm{N}_{n}(I)} & \bigotimes_{f_{i}=f^{\prime} \circ f^{\prime \prime}} A\left(i_{0}\right) \\ \bigotimes_{u \in \mathrm{N}_{n}(I)} & \bigotimes_{g: i_{-1} \leftarrow i_{0}} A\left(i_{-1}\right) \\ \bigotimes_{u \in \mathrm{N}_{n}(I)} & \bigotimes_{h: i_{n} \leftarrow i_{n+1}} A\left(i_{0}\right) .\end{cases}
$$

The faces $\partial_{i}$ are defined using these isomorphisms and the multiplicative structure:

$$
\begin{aligned}
\partial_{0} & =\bigotimes_{u \in \mathrm{N}_{n}(I)}\left(\bigotimes_{g: i_{-1} \leftarrow i_{0}} A\left(i_{-1}\right) \stackrel{\Psi_{u}}{\longrightarrow} A\left(i_{0}\right)\right), \\
\partial_{i} & =\bigotimes_{u \in \mathrm{N}_{n}(I)}\left(\bigotimes_{f_{i}=f^{\prime} \circ f^{\prime \prime}} A\left(i_{0}\right) \stackrel{\text { mult }}{\longrightarrow} A\left(i_{0}\right)\right), \quad 1 \leq i \leq n, \\
\partial_{n+1} & =\bigotimes_{u \in N_{n}(I)}\left(\bigotimes_{h: i_{n} \leftarrow i_{n+1}} A\left(i_{0}\right) \stackrel{\text { mult }}{\longrightarrow} A\left(i_{0}\right)\right),
\end{aligned}
$$

where $\Psi_{u}\left(\otimes_{g} x_{g}\right)$ is the product of the $A(g)\left(x_{g}\right)$ over the finite set of maps $g: i_{-1} \leftarrow i_{0}$ with source $i_{0}$, for $x_{g} \in A\left(i_{-1}\right)$. To define the codegeneracies $\sigma_{j}$ we use the unit map $\eta: \mathbb{K} \rightarrow A(i)$ associated to any CDGA and we set

$$
\sigma_{j}: \bigotimes_{u^{\prime} \in \mathrm{N}_{n-1}(I)} A\left(i_{0}^{\prime}\right) \cong \bigotimes_{u \in \mathrm{N}_{n}(I), f_{j}=\mathrm{id}} A\left(i_{0}\right) \stackrel{\mathrm{id} \otimes \eta}{\longrightarrow} \bigotimes_{u \in \mathrm{N}_{n}(I)} A\left(i_{0}\right) .
$$

We call $\otimes_{\bullet} A$ the simplicial replacement of the contravariant functor $A$. Notice that we need the nerve of $I$ to be finite in each degree for the above maps to be well defined.

When $F: I \rightarrow$ Top is a formal diagram of spaces, applying this simplicial replacement to each term in the zigzag of CDGA quasi-isomorphisms connecting $A_{\mathrm{PL}}(F)$ and $\mathrm{H}^{*}(F)$ gives a zigzag of quasi-isomorphisms of simplicial CDGAs

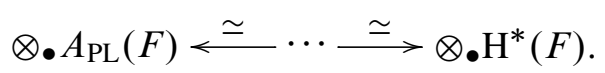

Kunneth (quasi-)isomorphisms induce a quasi-isomorphism of simplicial CDGAs

$$
\otimes_{\bullet} A_{\mathrm{PL}}(F) \stackrel{\simeq}{\longrightarrow} A_{\mathrm{PL}}\left(\Pi^{\bullet} F\right),
$$

and similarly the standard Kunneth isomorphism gives an isomorphism

$$
\otimes \bullet \mathrm{H}^{*}(F) \stackrel{\cong}{\longrightarrow} \mathrm{H}^{*}\left(\Pi^{\bullet} F\right)
$$

Combining this with the quasi-isomorphisms (3-1) proves the formality of $\Pi^{\bullet} F$. 


\section{Convergence of Bousfield-Kan spectral sequences for cosimplicial spaces and their truncations}

Throughout this section, $X^{\bullet}$ is a simply connected pointed cosimplicial space. We will establish a condition on such a cosimplicial space which guarantees that the BousfieldKan spectral sequences of its truncations converge. We also prove a folklore theorem which says that a left cofinal change of shape preserves the $E^{2}$ pages of the homotopy and homology Bousfield-Kan spectral sequences.

Our main convergence hypotheses will be the following:

Definition 4.1 We say that $X^{\bullet}$ is well above the diagonal at page $E^{r}$ if that page of the second-quadrant homotopy Bousfield-Kan spectral sequence associated to $X^{\bullet}$ satisfies the conditions

(i) $E_{-p, q}^{r}=0$ for $q \leq p$, and

(ii) for each $i$ there are finitely many $p$ such that $E_{-p, p+i}^{r} \neq 0$.

In other words, these conditions say that there are no terms on the main diagonal $E_{-p, p}^{r}$ or below, and that there are finitely many terms on each diagonal line of slope -1 . Such conditions already appear in [5].

Consider now the $n$-th truncation $X_{[n]}$ of $X^{\bullet}$. To prove a convergence result for the Bousfield-Kan spectral sequences of its cosimplicial replacement $\Pi^{\bullet}\left(X_{[n]}\right)$, we need the following lemma relating the homotopy spectral sequences associated to $X^{\bullet}$ and $\Pi^{\bullet}\left(X_{[n]}\right)$.

Recall the conormalization functor $\mathrm{N}^{*}: \mathrm{Ab}^{\Delta} \rightarrow \mathrm{Ch}^{\geq 0}$ from cosimplicial abelian groups to nonnegatively graded cochain complexes as defined in [39, Section 8.4] or in [5, Section 2]. For a cochain complex $C^{*}$ define its $n-$ th truncation $\tau^{n} C^{*}$ to be the cochain complex given by

$$
\begin{cases}\left(\tau^{n} C^{*}\right)^{q}=C^{q} & \text { if } q \leq n, \\ \left(\tau^{n} C^{*}\right)^{q}=0 & \text { if } q>n,\end{cases}
$$

with the differential induced in the obvious way from the differential on $C^{*}$.

Lemma 4.2 The $E^{2}$ page of the homotopy Bousfield-Kan spectral sequence associated to $\Pi^{\bullet}\left(X_{[n]}\right)$ is given by

$$
E_{-p, q}^{2}=\mathrm{H}^{p}\left(\tau^{n} \mathrm{~N}^{*}\left(\pi_{q}\left(X^{\bullet}\right)\right)\right) .
$$


Proof The following proof was given to us by P Bousfield. By [6, XI.7.1] we have an isomorphism $E_{-p, q}^{2} \cong \lim _{\Delta[n]}^{p} \pi_{q}\left(X_{[n]}\right)$, where $\lim ^{p}$ is the $p$-th left derived functor of $\lim ^{0}=\lim$. Recall for example from [39, 8.4] the Dold-Kan Theorem which states that the conormalization functor $\mathrm{N}^{*}: \mathrm{Ab}^{\Delta} \rightarrow \mathrm{Ch}^{\geq 0}$ is an equivalence of abelian categories. It is not difficult to adapt the proof of that result to get an equivalence of abelian categories

$$
\mathrm{N}_{n}^{*}: \mathrm{Ab}^{\Delta[n]} \longrightarrow \mathrm{Ch}^{[n]}
$$

between $n$-truncated cosimplicial abelian groups and cochain complexes concentrated in degrees $0, \ldots, n$. Here $\mathrm{N}_{n}^{*}$ is defined in the obvious way by mimicking the definition of the usual conormalization $\mathrm{N}^{*}$. In particular for a cosimplicial abelian group $A^{\bullet}$ we have that $\mathrm{N}_{n}^{*}\left(A_{[n]}\right) \cong \tau^{n}\left(\mathrm{~N}^{*}\left(A^{\bullet}\right)\right)$.

Notice that, as with the usual Dold-Kan correspondence, for a truncated cosimplicial abelian group $A$ in $\mathrm{Ab}^{\Delta[n]}$ we have an isomorphism $\lim _{\Delta[n]}^{0} A \cong \mathrm{H}^{0}\left(\mathrm{~N}_{n}^{*}(A)\right)$. By a universal $\delta$-functor argument, $\mathrm{H}^{p}$ is the $p$-th left derived functor of $\mathrm{H}^{0}$ in $\mathrm{Ch}^{[n]}$. The equivalence of abelian categories implies therefore that $\lim _{\Delta[n]}^{p} A \cong \mathrm{H}^{p}\left(\mathrm{~N}_{n}^{*}(A)\right)$. Collecting these results proves our lemma.

Proposition 4.3 If $X^{\bullet}$ is well above the diagonal at the $E^{1}$ page then

(i) the homotopy and homology Bousfield-Kan spectral sequences associated to $X^{\bullet}$ converge strongly to $\pi_{*}\left(\right.$ hoTot $\left.X^{\bullet}\right)$ and $\mathrm{H}_{*}\left(\right.$ hoTot $\left.X^{\bullet}\right)$;

(ii) the homotopy and homology Bousfield-Kan spectral sequences associated to $\Pi^{\bullet}\left(X_{[n]}\right)$ converge strongly to $\pi_{*}\left(\operatorname{hoTot}^{n} X^{\bullet}\right)$ and $\mathrm{H}_{*}\left(\operatorname{hoTot}^{n} X^{\bullet}\right)$;

(iii) $\mathrm{H}_{*}\left(\operatorname{hoTot} X^{\bullet}\right) \cong \lim _{*} \mathrm{H}_{*}\left(\operatorname{hoTot}^{n} X^{\bullet}\right)$.

Proof (i) Since $X^{\bullet}$ is well above the diagonal at $E^{1}$ it is also well above the diagonal at $E^{2}$. Then the statement we want is exactly the content of the results in [6, Proposition IX.5.7] and [5, Theorem 3.2].

(ii) By Lemma 4.2 we have that $\Pi^{\bullet}\left(X_{[n]}\right)$ is well above the diagonal at $E^{2}$. Moreover $\operatorname{hoTot}\left(\Pi^{\bullet}\left(X_{[n]}\right)\right) \simeq \operatorname{hoTot}^{n} X^{\bullet}$. Thus (ii) follows from the same argument as in (i).

(iii) By the hypothesis and Lemma 4.2 we have that the connectivity of the map of spectral sequences between the $E^{2}$ pages of the homotopy spectral sequence for $X^{\bullet}$ and $\Pi^{\bullet}\left(X_{[n]}\right)$ tends to infinity with $n$. By the convergence of these homotopy spectral sequences this implies that the connectivity of the map hoTot $X^{\bullet} \rightarrow \operatorname{hoTot}^{n} X^{\bullet}$ tends to infinity with $n$. Therefore the same is true for the homologies.

The following is part of the content of [31, Corollary 7.4]. 
Proposition 4.4 (Sinha) For $d \geq 4, \mathcal{K}_{d}^{\bullet}$ is well above the diagonal at the $E^{1}$ page.

The last result of this section is a comparison theorem for Bousfield-Kan spectral sequences of cosimplicial replacements of diagrams which are connected by a left cofinal functor (see end of Section 2.1). We could not find a proof for this folklore result in the literature so we include one here. The proof below is due to W Dwyer (P Bousfield has also given us another proof).

Proposition 4.5 Let $\theta: I \rightarrow J$ be a functor between finite categories and let $F: J \rightarrow$ Top be a $J$-diagram of (pointed) spaces. If $\theta$ is left cofinal then both homotopy and rational homology Bousfield-Kan spectral sequences associated to the cosimplicial replacements $\Pi^{\bullet} F$ and $\Pi^{\bullet}\left(\theta^{*} F\right)$ agree from the $E^{2}$ pages.

Proof The proof mimics that of [6, Proposition XI.9.2] and we follow most of the notation from there. Recall from the proof of Proposition 3.3 the simplicial CDGA associated to a contravariant functor of CDGAs. The Kunneth quasi-isomorphism

$$
\otimes \cdot A_{\mathrm{PL}}(F) \stackrel{\simeq}{\longrightarrow} A_{\mathrm{PL}}\left(\Pi^{\bullet} F\right)
$$

implies that the rational homology Bousfield-Kan spectral sequence for $\Pi^{\bullet} F$ coincides from the $E_{2}$ term with the spectral sequence of the double complex $\mathrm{N}_{*}\left(\otimes_{\bullet} A_{\mathrm{PL}}(F)\right)$, and we have an analogous result for $\Pi^{\bullet} \theta^{*}(F)$.

Define a bisimplicial commutative graded algebra (CGA)

$$
\otimes_{\bullet, \bullet}\left(\mathrm{H}^{*}(F), \theta\right)
$$

with the $(n, q)$ term defined by

$$
\otimes_{n, q}\left(\mathrm{H}^{*}(F), \theta\right)=\otimes_{(u, v, \gamma)} \mathrm{H}^{*}\left(F\left(j_{0}\right)\right)
$$

where the tensor product is taken over

$$
u=\left(i_{0} \stackrel{\alpha_{1}}{\leftarrow} \ldots \stackrel{\alpha_{n}}{\leftarrow} i_{n}\right) \in \mathrm{N}_{n}(I), \quad v=\left(j_{0} \stackrel{\beta_{1}}{\leftarrow} \ldots \stackrel{\beta_{q}}{\leftarrow} j_{q}\right) \in \mathrm{N}_{q}(J), \quad \gamma: \theta\left(i_{0}\right) \rightarrow j_{q} .
$$

The faces and degeneracies in both directions are obvious generalizations of those in the proof of Proposition 3.3.

We can take the normalization of this bisimplicial CGA with respect to the first $(i=1)$ or the second $(i=2)$ simplicial degree to get a simplicial chain complex

$$
\mathrm{N}_{*}^{(i)}\left(\otimes_{\bullet, \bullet}\left(\mathrm{H}^{*}(F), \theta\right)\right) \text {. }
$$


In the first direction we have

$$
\left(\mathrm{N}_{*}^{(1)}\left(\otimes_{\bullet, \bullet}\left(\mathrm{H}^{*}(F), \theta\right)\right)\right)_{q}=\otimes_{v \in \mathrm{N}_{q}(J)} \mathrm{N}_{*}\left(\otimes_{(u, \gamma) \in \mathrm{N}_{\bullet}\left(F \downarrow j_{q}\right)} \mathrm{H}^{*}\left(F\left(j_{0}\right)\right)\right) .
$$

Denote by $\mathrm{H}^{*}\left(F\left(j_{0}\right)\right)$ • the constant cosimplicial CGA which consists of the ring $\mathrm{H}^{*}\left(F\left(j_{0}\right)\right)$ in each degree with the identity maps as faces and degeneracies. The category of simplicial CGAs is a simplicial model category [14, II.3 and II.5.2.(3)]. So we have the simplicial CGA

$$
\mathrm{N}_{\bullet}\left(F \downarrow j_{q}\right) \otimes \mathrm{H}^{*}\left(F\left(j_{0}\right)\right) \bullet \cong \otimes_{(u, \gamma) \in \mathrm{N}_{\bullet}\left(F \downarrow j_{q}\right)} \mathrm{H}^{*}\left(F\left(j_{0}\right),\right.
$$

and by cofinality, $\mathrm{N}_{\bullet}\left(F \downarrow j_{q}\right)$ is weakly equivalent to the simplicial set $*_{\bullet}$ consisting of the singleton in each degree. Using [14, Proposition II.3.4.] we get a weak equivalence of simplicial CGAs

$$
\mathrm{N}_{\bullet}\left(F \downarrow j_{q}\right) \otimes \mathrm{H}^{*}\left(F\left(j_{0}\right)\right) \bullet \simeq *_{\bullet} \otimes \mathrm{H}^{*}\left(F\left(j_{0}\right)\right) \bullet=\mathrm{H}^{*}\left(F\left(j_{0}\right)\right) \bullet
$$

and deduce a weak equivalence of simplicial chain complexes

$$
\mathrm{N}_{*}^{(1)}\left(\otimes_{\bullet, \bullet}\left(\mathrm{H}^{*}(F), \theta\right)\right) \simeq \otimes_{\bullet} \mathrm{H}^{*}(F)
$$

where each $\mathrm{H}^{*}\left(F\left(j_{0}\right)\right)$ is a chain complex concentrated in degree 0 .

In the other direction,

$$
\left(\mathrm{N}_{*}^{(2)}\left(\otimes_{\bullet, \bullet}\left(\mathrm{H}^{*}(F), \theta\right)\right)\right)_{n}=\otimes_{u \in \mathrm{N}_{n}(I)} \mathrm{N}_{*}\left(\otimes_{\bullet} \mathrm{H}^{*}\left(\theta\left(i_{0}\right) \downarrow F\right)\right),
$$

where $\theta\left(i_{0}\right) \downarrow F$ is the composite

$$
\theta\left(i_{0}\right) \downarrow J \longrightarrow J \stackrel{F}{\longrightarrow} \text { Top. }
$$

Here $\theta\left(i_{0}\right) \downarrow J$ is the undercategory (denoted by $J \backslash \theta\left(i_{0}\right)$ in [6]). This undercategory has the identity map at $\theta\left(i_{0}\right)$ as an initial object. It is easy to deduce, by an extra degeneracy or a spectral sequence argument, that $\otimes_{\bullet} \mathrm{H}^{*}\left(\theta\left(i_{0}\right) \downarrow F\right)$ is weakly equivalent to the constant simplicial CGA $\mathrm{H}\left(F\left(\theta\left(i_{0}\right)\right)\right.$. We then have a weak equivalence of simplicial chain complexes

$$
\mathrm{N}_{*}^{(2)}\left(\otimes_{\bullet, \bullet}\left(\mathrm{H}^{*}(F), \theta\right)\right) \simeq \otimes_{\bullet} \mathrm{H}^{*}\left(\theta^{*} F\right)
$$

where each $\mathrm{H}^{*}\left(\theta^{*} F\left(i_{0}\right)\right)$ is a chain complex concentrated in degree 0 .

The left hand sides of the weak equivalences (4-1) and (4-2) are the $E_{1}$ page of spectral sequences computing the homology of the totalization of the double complex obtained as the double normalization of $\otimes_{\bullet, \bullet}\left(\mathrm{H}^{*}(F), \theta\right)$. Moreover these weak equivalences show that the homology of the $E_{1}$ pages is concentrated on a single line. Hence both of these spectral sequences collapse at $E_{2}$, and since they converge to the same thing, 
these $E_{2}$ pages are isomorphic. We deduce that the homologies of the normalizations of $\otimes_{\bullet} \mathrm{H}^{*}(F)$ and $\otimes_{\bullet} \mathrm{H}^{*}\left(\theta^{*} F\right)$ are isomorphic. Those are exactly the $E_{2}$ pages of the homology Bousfield Kan spectral sequences of $\Pi^{\bullet} F$ and $\Pi^{\bullet} \theta^{*} F$.

The proof for the homotopy spectral sequence is similar.

\section{Categories of fans, fanic diagrams and truncated cosimpli- cial objects}

In this section we introduce a sequence of finite categories $\Phi[n]$ that we call categories of $n$-fans. They will serve in the next section as shapes of certain fanic diagrams associated to morphisms of operads, generalizing the Gerstenhaber-Voronov cosimplicial object associated to a multiplicative operad. We also construct a left cofinal functor $\phi_{n}: \Phi[n] \rightarrow \Delta[n]$.

Recall that a planar tree is an isotopy class of an embedding of the realization of a contractible finite 1-dimensional simplicial complex in the plane. In particular a planar tree consists of a finite set of vertices and edges. The valence of a vertex is the number of edges ending in that vertex. A leaf is a vertex of valence 1 . The embedding in the plane induces a clockwise cyclic order on the leaves.

Definition 5.1 - For a natural number $n$, an $n-f a n$ is a planar tree with a distinguished vertex called the bead such that each vertex, except maybe the bead, is of valence different from 2 and with $n+1$ leaves other than the bead which are labeled in the clockwise cyclic order by $0,1, \ldots, n$. The leaf labeled 0 is called the root. The bead and vertices which are not leaves are called the nonlabeled vertices.

- Define a partial order on the set of $n$-fans by declaring that $T \leq T^{\prime}$ if the $n$-fan $T^{\prime}$ is obtained from the $n$-fan $T$ by contracting some edges connecting nonlabeled vertices and where the bead in $T^{\prime}$ is the vertex obtained by contracting the subtree of $T$ containing its bead.

- The category corresponding to such a poset of $n$-fans is called the $n$-fanic category and is denoted by $\Phi[n]$. Thus there is a unique morphism $T \rightarrow T^{\prime}$ if and only if $T \leq T^{\prime}$.

- A diagram shaped on the fanic category $\Phi[n]$ is called an $n$-fanic diagram.

Example 5.2 Figure 1 gives examples of an 8-fan in (a) and of two 3 -fans in (b) and (c). The bead is the vertex pictured as a small circle. The left-hand side of Figure 2 represents the category $\Phi[2]$. 


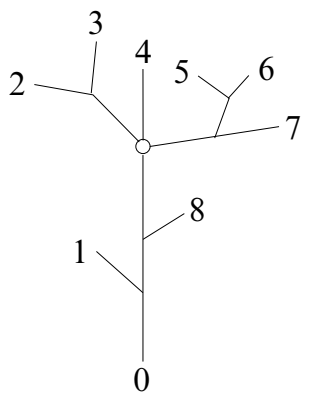

(a)

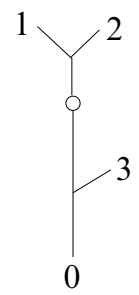

(b)

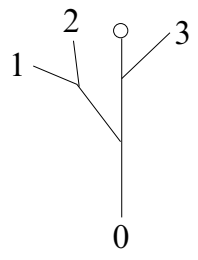

(c)

Figure 1: Three examples of fans

Remark 5.3 The name "fan" is motivated by the fact that one can define a functor from fans to rooted trees which "opens" them along the edge emanating from the bead, much as one opens a fan. For more details on this, see our paper [21]. The realization of $\Phi[n]$ is homeomorphic to the barycentric subdivision of the $n$-dimensional cyclohedron $C_{n}$ introduced by Bott and Taubes in [4].

Definition 5.4 We say that a $n$-fan $T$ is $i$-separated for $i \in[n]$ if either

- the bead is not a leaf and the shortest path in $T$ joining leaves $i$ and $(i+1)$ mod $(n+1)$ goes through the bead, or

- the bead is a leaf and it is between leaves $i$ and $(i+1) \bmod (n+1)$ in the clockwise cyclic order.

Example 5.5 In Example 5.2 the fans are $i$-separated for

(a) $i=1,3,4,7$;

(b) $i=0,2$;

(c) $i=2$.

Let $\mathcal{P}_{0}([n])$ be the category whose objects are nonempty subsets of $[n]=\{0, \ldots, n\}$ and whose morphisms are inclusions. Define a functor

$$
\theta_{n}: \Phi[n] \longrightarrow \mathcal{P}_{0}([n])
$$

by setting, for an $n$-fan $T$,

$$
\theta_{n}(T):=\{i \in[n]: T \text { is } i \text {-separated }\} .
$$

It is immediate that $\theta_{n}$ is an order-preserving map between the two posets and hence a functor. 
Proposition 5.6 The functor $\theta_{n}: \Phi[n] \longrightarrow \mathcal{P}_{0}([n])$ is left cofinal.

Proof A quick way to prove this result would be to write this functor as a composition $\theta_{n}=\mathcal{F}_{n} \Pi_{n}$, where $\Pi_{n}$ is the projection from the poset of faces of a cyclohedron to the poset of faces of an associahedron; see [21, Subsection 2.3] and Remark 5.3. The functor $\mathcal{F}_{n}$ is defined in [31, Definition 4.14]. By [21, Theorem 5] $\Pi_{n}$ is left cofinal, and by [31, Proposition 4.15] $\mathcal{F}_{n}$ has the same property. This would imply the cofinality of $\theta_{n}$.

However, to the best of our understanding, the proof of [31, Proposition 4.15] appear to be incorrect. Contrary to what is stated there, the poset $\mathcal{F}_{n} \downarrow S$ does not in general have a terminal object. ${ }^{1}$ Similarly $\theta_{n} \downarrow S$ does not in general have a terminal object.

However, here is another proof of the cofinality of $\theta_{n}$. The argument that follows in fact can be used in exactly the same way to show the cofinality of $\mathcal{F}_{n}$.

We will be using the following general result.

Lemma 5.7 [21, Proposition 3.13] Let $\Pi: B \rightarrow A$ be a map of posets satisfying the following properties:

- For any $a \in A$, the poset $\Pi^{-1}(a)$ is contractible.

- For any $a \in A$ and $b \in B$ such that $a \leq \Pi(b)$, the poset

$$
\Pi^{-1}(a \mid \leq b)=\left\{b^{\prime} \in B \mid \Pi\left(b^{\prime}\right)=a \text { and } b^{\prime} \leq b\right\}
$$

is contractible.

Then $\Pi$ is left cofinal.

It is easy to see that $\theta_{n}^{-1}(S)$ has a terminal element given by the only fan $T \in \Phi[n]$ such that $\theta_{n}(T)=S$ and the distance between the bead and any of its leaves is at most 2. Thus $\theta_{n}^{-1}(S)$ is contractible. (The morphisms in $\theta_{n}^{-1}(S)$ contract only those edges that are not adjacent to the bead. The poset $\theta_{n}^{-1}(S)$ is actually isomorphic to a product of posets with each factor being the poset of faces of an associahedron.) Similarly any poset $\theta_{n}^{-1}(S \mid \leq T)$ (such that $S \subset \theta_{n}(T)$ ) has a terminal element given by a fan $T^{\prime}$ obtained from $T$ by an appropriate expansion of the bead. ${ }^{2}$ For example,

\footnotetext{
${ }^{1}$ For example, the poset $\mathcal{F}_{3} \downarrow\{2,3\}$ does not have a terminal object (see [31, Figure 4.4]); its realization is the union of the lower and the left edges of the pentagon on that figure.

${ }^{2} \mathrm{By}$ "expansion of the bead" we mean a surgery on the fan that changes only the vicinity of the bead as in this figure:
}

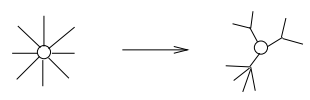


for a tree $T$

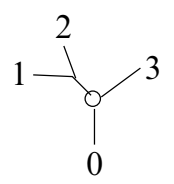

(notice that $\theta_{3}(T)=\{0,2,3\}$ ), the terminal object of $\theta_{3}^{-1}(\{2,3\} \mid \leq T\}$ is:

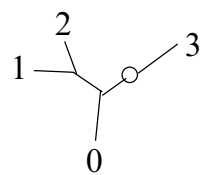

Recall the functor $\mathcal{G}_{n}: \mathcal{P}_{0}([n]) \rightarrow \Delta[n]$ from [31, Definition 6.3], defined as follows. For a nonempty subset $S \subset[n]$ consider the only order preserving bijection $f_{S}: S \stackrel{\cong}{\rightrightarrows}$ $[(\# S)-1]$. Define $\mathcal{G}_{n}(S)=[(\# S)-1]$ and for a morphism $j: S \hookrightarrow T$ in $\mathcal{P}_{0}([n])$ let $\mathcal{G}_{n}(j)$ be the composite $f_{T} \circ j \circ f_{S}^{-1}$. It turns out that $\mathcal{G}_{n}$ is left cofinal [31, Theorem 6.7].

Now let $\phi_{n}$ be the composite

$$
\phi_{n}:=\mathcal{G}_{n} \theta_{n}: \Phi[n] \longrightarrow \Delta[n] .
$$

Theorem 5.8 The functor $\phi_{n}: \Phi[n] \rightarrow \Delta[n]$ is left cofinal.

Proof This is immediate from Proposition 5.6 and the cofinality of $\mathcal{G}_{n}$ from [31, Theorem 6.7].

Example 5.9 Figure 2 gives the shapes of $\Phi[2], \mathcal{P}_{0}([2])$, and $\Delta[2]$ and the functors $\theta_{2}$ and $\mathcal{G}_{2}$.

\section{The fanic diagram associated to a morphism of nonsym- metric operads}

Recall from Section 2.2 the construction that associates a cosimplicial object to a multiplicative operad. Here we generalize this construction by associating a fanic diagram to any morphism of nonsymmetric operads.

Let $\mu: \mathcal{R} \rightarrow \mathcal{M}$ be a morphism of nonsymmetric operads in a symmetric monoidal category $\mathcal{C}$. Recall the category $\Phi[n]$ of $n$-fans defined in Section 5. We now describe a fanic diagram

$$
\widehat{\mathcal{M}}_{\{n\}}: \Phi[n] \longrightarrow \mathcal{C}
$$




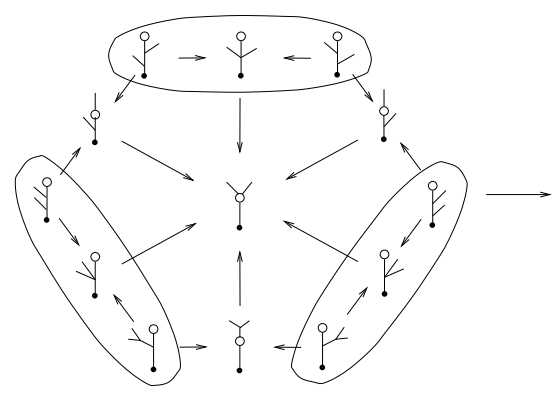

$\Phi[2]$

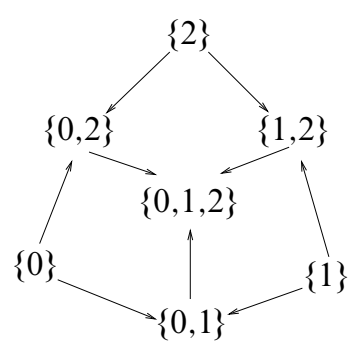

$\mathcal{P}_{0}([2])$
$[0] \doteq[1] \doteq[2]$

$\Delta[2]$

Figure 2: The functor $\phi_{2}$

which will depend on $\mu$ even if only $\mathcal{M}$ appears in the notation. We first define the value of the diagram $\widehat{\mathcal{M}}_{\{n\}}$ on objects. Let $T \in \Phi[n]$ be an $n$-fan. Orient each edge of $T$ in the unique way such that its origin is on the shortest path joining the root to the end of that edge. For a vertex $v$ other than the root let $|v|$ be the number of edges emanating from $v$. Since $v$ has exactly one incoming edge, we have $|v|=$ valence $(v)-1$.

Recall that the leaves other than the bead of the fan $T$ are labeled and that the other vertices, including the bead, are called the nonlabeled vertices. For any nonlabeled vertex $v$ of $T$ set

Set

$$
\mathcal{M}(T: v):= \begin{cases}\mathcal{M}(|v|) & \text { if } v \text { is the bead, } \\ \mathcal{R}(|v|) & \text { if } v \text { is not the bead. }\end{cases}
$$

$$
\widehat{\mathcal{M}}_{\{n\}}(T):=\bigotimes_{v} \mathcal{M}(T: v)
$$

where the monoidal product is taken over all the nonlabeled vertices $v$ of $T$.

To define $\widehat{\mathcal{M}}_{\{n\}}$ on morphisms, let $e$ be an edge of $T$ between two nonlabeled vertices $x$ and $y$ such that $e$ is oriented from $x$ to $y$. Since $x$ is not the root there is a single edge that ends in $x$. Label this edge 0 and label all the edges emanating from $x$ with $1, \ldots,|x|$ such that they appear in the clockwise order. Hence the edge $e$ is assigned some label $1 \leq i \leq|x|$. Let $\bar{T}:=T / e$ be the fan obtained by contracting the edge $e$ and let $\bar{e}$ be the vertex in $\bar{T}$ corresponding to that contracted edge. By definition, $\bar{e}$ is the bead of $\bar{T}$ if and only if $x$ or $y$ is the bead of $T$. Notice also that the nonlabeled vertices $v$ other than $x$ and $y$ in $T$ are in bijection with the nonlabeled vertices of $\bar{T}$ other than $\bar{e}$. The operadic structures and the morphism $\mu$ induce an 
obvious insertion map

$$
\circ_{i}: \mathcal{M}(T: x) \otimes \mathcal{M}(T: y) \longrightarrow \mathcal{M}(\bar{T}: \bar{e}) .
$$

We define the morphism $\widehat{\mathcal{M}}_{\{n\}}(T \rightarrow T / e)$ to be the composite

$$
\begin{aligned}
\widehat{\mathcal{M}}_{\{n\}}(T) \cong \mathcal{M}(T: x) \otimes \mathcal{M}(T: y) \otimes & \bigotimes_{v \neq x, y} \mathcal{M}(T: v) \\
& \stackrel{\circ_{i} \otimes \mathrm{id}}{\longrightarrow} \mathcal{M}(\bar{T}: \bar{e}) \otimes \bigotimes_{v \neq \bar{e}} \mathcal{M}(\bar{T}: v) \cong \widehat{\mathcal{M}}_{\{n\}}(\bar{T}) .
\end{aligned}
$$

Proposition 6.1 Let $n \geq 0$ and let $\mathcal{C}$ be a symmetric monoidal category.

(1) For any morphism $\mu: \mathcal{R} \rightarrow \mathcal{M}$ of nonsymmetric operads in $\mathcal{C}$ the above construction gives a well-defined functor

$$
\widehat{\mathcal{M}}_{\{n\}}: \Phi[n] \longrightarrow \mathcal{C} .
$$

(2) This construction is functorial, ie any commutative square of nonsymmetric operads

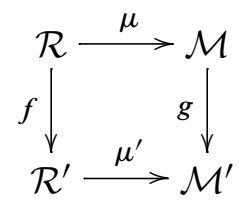

induces a natural transformation $\widehat{g}: \widehat{\mathcal{M}}_{\{n\}} \longrightarrow \widehat{\mathcal{M}}_{\{n\}}^{\prime}$.

Proof For (1), we have already defined $\widehat{\mathcal{M}}_{\{n\}}$ on objects and contractions of one edge. Since any morphism in $\Phi[n]$ is a composition of such contractions, we only have to check that the image under $\widehat{\mathcal{M}}_{\{n\}}$ of a composition does not depend on the order in which we contract the edges. For this, it is enough to check that if $e_{1}$ and $e_{2}$ are two distinct edges between nonlabeled vertices in an $n$-fan $T$, then

$$
\begin{aligned}
\widehat{\mathcal{M}}_{\{n\}}\left(T / e_{1} \rightarrow T /\left\{e_{1}, e_{2}\right\}\right) \circ \widehat{\mathcal{M}}_{\{n\}} & \left(T \rightarrow T / e_{1}\right) \\
= & \widehat{\mathcal{M}}_{\{n\}}\left(T / e_{2} \rightarrow T /\left\{e_{1}, e_{2}\right\}\right) \circ \widehat{\mathcal{M}}_{\{n\}}\left(T \rightarrow T / e_{2}\right) .
\end{aligned}
$$

This is an elementary check and is left to the reader.

For (2), given an object $T$ of $\Phi[n]$, set $\hat{g}(T):=\bigotimes_{v} \hat{g}(T: v)$ where the tensor product is taken over the nonlabeled vertices $v$ of $T$ and

$$
\hat{g}(T: v):= \begin{cases}g(|v|) & \text { if } v \text { is the bead, } \\ f(|v|) & \text { if } v \text { is not the bead. }\end{cases}
$$


This defines the desired natural transformation.

Suppose moreover that the category $\mathcal{C}$ is equipped with a certain class of morphisms called weak equivalences. This induces a class of weak equivalences on diagrams in $\mathcal{C}$ by declaring that a natural transformation between two diagrams is a weak equivalence if the map associated to each object of the indexing category is a weak equivalence. In particular this induces a class of weak equivalences of operads in $\mathcal{C}$.

Proposition 6.2 Suppose that the symmetric monoidal category is equipped with a class of morphisms called weak equivalences that is stable under $\otimes$ and contains all isomorphisms. If the morphisms of nonsymmetric operads $f$ and $g$ in the commutative square (6-1) are weak equivalences then the natural transformation $\hat{g}$ is also a weak equivalence.

Proof Follows from the definition of $\hat{g}$ and the fact that the class of weak equivalences is stable under tensor product and contains the reordering isomorphisms.

Lastly, we explain in which sense the above construction is a generalization of the cosimplicial object associated to a multiplicative nonsymmetric operad. Recall the functor $\phi_{n}: \Phi[n] \rightarrow \Delta[n]$ from Equation (5-1) which is left cofinal by Theorem 5.8. The proof of the following is straightforward.

Theorem 6.3 Let $\mu: \mathcal{A S S} \rightarrow \mathcal{M}$ be a multiplicative nonsymmetric operad. Let $\mathcal{M}_{[n]}: \Delta[n] \rightarrow \mathcal{C}$ be the $n-t h$ truncation of the associated cosimplicial object $\mathcal{M}^{\bullet}$ and let $\widehat{\mathcal{M}}_{\{n\}}$ be the $n$-fanic diagram associated to the morphism $\mu$. Then the following diagram commutes:

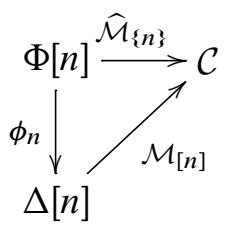

Corollary 6.4 Let $\mathcal{M}$ be a multiplicative operad of spaces, let $\mathcal{M}^{\bullet}$ be the associated cosimplicial space and let $\widehat{\mathcal{M}}_{\{n\}}$ be the associated $n$-fanic diagram for $n \geq 0$. Then there is a homotopy equivalence

$$
\underset{\Phi[n]}{\operatorname{holim}} \widehat{\mathcal{M}}_{\{n\}} \simeq \operatorname{hoTot}^{n}\left(\mathcal{M}^{\bullet}\right) .
$$

Proof This is a consequence of Theorem 6.3 and Theorem 5.8. 
Remark 6.5 Notice that all the results of this section can be easily dualized for cooperads. In particular, to a morphism of cooperads $\mu^{*}: \mathcal{M}^{*} \rightarrow \mathcal{R}^{*}$ in $\mathcal{C}$ we can associate a cofanic diagram $\widehat{\mathcal{M}}_{\{n\}}: \Phi[n]^{\text {op }} \rightarrow \mathcal{C}$ where $(-)^{\text {op }}$ means the opposite category. We leave it as an exercise for the reader to state and prove these dual results.

Remark 6.6 Notice that the map $\mu: \mathcal{R} \rightarrow \mathcal{M}$ endows $\mathcal{M}$ with the structure of a bimodule over the nonsymmetric operad $\mathcal{R}$. It is easy to see that the entire discussion above can actually be applied to any such bimodule. In particular, one has an associated fanic diagram for a bimodule (this motivates the notation $\widehat{\mathcal{M}}_{\{n\}}$ where $\mathcal{M}$ denotes that bimodule).

Remark 6.7 It is possible to describe an infinite category $\Phi$ filtered by the categories $\Phi[n]$. It is also possible to construct an $\infty$-fanic diagram $\widehat{\mathcal{M}}_{\{\infty\}}: \Phi \rightarrow \mathcal{C}$ whose restriction to $\Phi[n]$ is $\widehat{\mathcal{M}}_{\{n\}}$, and a left cofinal functor $\phi: \Phi \rightarrow \Delta$. In the case of a multiplicative operad $\mathcal{M}$, we have $\phi^{*} \mathcal{M}^{\bullet} \cong \widehat{\mathcal{M}}_{\{\infty\}}$. This seems to be a more natural generalization of the Gerstenhaber-Voronov construction since it relates directly to the cosimplicial object $\mathcal{M}^{\bullet}$ instead of its truncations. However, we do not use this construction here since the homology Bousfield-Kan spectral sequence of the cosimplicial replacement of the infinite category $\Phi$ might be troublesome.

\section{Formality of the Fulton-MacPherson fanic diagram}

Recall from Section 2.4 the Fulton-MacPherson operad $\mathcal{F}_{d}$ which consists of suitable compactifications of configuration spaces in $\mathbb{R}^{d}$. A linear inclusion $\epsilon: \mathbb{R} \rightarrow \mathbb{R}^{d}$ induces a morphism of nonsymmetric operads

$$
\epsilon_{\#}: \mathcal{F}_{1}^{(0)} \rightarrow \mathcal{F}_{d}
$$

where $\mathcal{F}_{1}^{(0)}$ is the principal path component of the Fulton-MacPherson operad in dimension 1. From Section 6, we then have an associated $n$-fanic Fulton-MacPherson diagram

$$
\widehat{\mathcal{F}}_{d\{n\}}: \Phi[n] \longrightarrow \text { Top. }
$$

The goal of this section is to establish the following:

Theorem 7.1 For $d \geq 3$ and $n \geq 0$, the Fulton-MacPherson fanic diagram $\widehat{\mathcal{F}}_{d\{n\}}$ is $\mathbb{R}$-formal.

The proof of this theorem is based on Kontsevich's theorem on the formality of the little $d$-disks operad, proved in [18, Section 3], with some relevant parts appearing in 
[19, Appendix 8]. A more detailed proof can be found in [22]. We now recall the main ingredients and ideas of this proof.

To prove the formality of the little $d$-disks operad, Kontsevich proves the formality of $\mathcal{F}_{d}$, the homotopy equivalent Fulton-MacPherson operad in dimension $d$ (even if in [18] he defines what is now called the Kontsevich operad). To do so he starts by constructing a combinatorial cooperad of CDGAs as follows.

Consider the set of finite oriented graphs with $n$ external vertices (labeled from 1 to $n$ ) and some other, internal, vertices. Each internal vertex is at least trivalent and is connected by a path to some external vertex. No double edges or loops are allowed and an ordering of the internal vertices and edges is imposed. Such graphs are called admissible [18, Definition 13]. Denote by $\mathcal{D}_{d}(n)$ the real vector space generated by admissible graphs with $n$ external vertices, and with certain identifications (with appropriate signs) having to do with reordering of internal vertices or edges or reversal of orientations of edges. The degree of an admissible graph $\Gamma$ is $\operatorname{deg}(\Gamma):=e(d-1)-q d$ where $e$ is the number of edges and $q$ is the number of internal vertices. A degree +1 differential $\mathrm{d}(\Gamma)$ on $\mathcal{D}_{d}(n)$ is defined as the alternating sum of the graphs obtained from $\Gamma$ by contracting an edge whose at least one vertex is internal. There is also a multiplication on $\mathcal{D}_{d}(n)$ where the product of two admissible graphs is obtained by gluing the graphs along their common external vertices. This equips $\mathcal{D}_{d}(n)$ with the structure of a CDGA over $\mathbb{R}$. Moreover, the sequence $\mathcal{D}_{d}=\left\{\mathcal{D}_{d}(n)\right\}_{n \geq 0}$ admits a structure of a cooperad in CDGA.

One other important ingredient, developed in [19, Appendix 8], is the functor of semialgebraic differential forms

$$
\Omega_{\mathrm{PA}}^{*}:\{\text { semialgebraic sets }\} \longrightarrow \text { CDGA, }
$$

mimicking the de Rham functor of smooth differential forms on smooth manifolds, where a semialgebraic set is a subset of $\mathbb{R}^{n}$ defined by finite sets of polynomial inequalities and boolean operations. This functor $\Omega_{\mathrm{PA}}^{*}$ is naturally quasi-isomorphic to the functor $A_{\mathrm{PL}}(-; \mathbb{R})$. One fact which will be important to us is that if $X$ is a semialgebraic set of dimension $\operatorname{dim}(X) \leq m$ then $\Omega_{\mathrm{PA}}^{i}(X)=0$ for $i>m$.

The spaces $\mathcal{F}_{d}(n)$ are semialgebraic manifolds and Kontsevich's idea is to assign to each admissible graph $\Gamma \in \mathcal{D}_{d}(n)$ of degree $r$ a certain semialgebraic differential form $I(\Gamma)=\omega_{\Gamma} \in \Omega_{\mathrm{PA}}^{r}\left(\mathcal{F}_{d}(n)\right)$. This defines a CDGA morphism

$$
I_{n}: \mathcal{D}_{d}(n) \longrightarrow \Omega_{\mathrm{PA}}^{*}\left(\mathcal{F}_{d}(n)\right) .
$$

On the other hand, it is easy to construct an explicit CDGA morphism

$$
\bar{I}_{n}: \mathcal{D}_{d}(n) \longrightarrow \mathrm{H}^{*}\left(\mathcal{F}_{d}(n) ; \mathbb{R}\right)
$$


that sends to 0 any admissible graphs with at least one internal vertex and that sends the admissible graph with a unique edge joining the external vertices $i$ and $j$, for $1 \leq i<j \leq n$, to the generator $g_{i j}$ in the usual presentation of the cohomology of the configuration space

$$
\mathrm{H}^{*}\left(\mathcal{F}_{d}(n) ; \mathbb{R}\right) \cong \bigwedge\left(\left\{g_{i j}: 1 \leq i<j \leq n\right\}\right) / \sim .
$$

The exact presentation for the three equivalence relations $\sim$ (one of which is the Arnold, or three-term, relation) can be found, for example, in [31, Section 7].

It can be proved that $\bar{I}_{n}$ is a quasi-isomorphism and, since $I_{n}$ is surjective on the indecomposables in cohomology, it is also a quasi-isomorphism. Finally, it would be nice if these were quasi-isomorphisms of cooperads, but they are not because the contravariant functor $\Omega_{\mathrm{PA}}^{*}$ is not monoidal and so $\Omega_{\mathrm{PA}}^{*}\left(\mathcal{F}_{d}\right)$ does not inherit the structure of a cooperad. Nevertheless $I=\left\{I_{n}\right\}_{n \geq 0}$ is almost a quasi-isomorphism of cooperads. More precisely, we have the following:

Theorem 7.2 [22] There is a commutative diagram

$$
\begin{gathered}
\mathcal{D}_{d}(n) \stackrel{\phi}{\longrightarrow} \mathcal{D}_{d}(k) \otimes \mathcal{D}_{d}\left(n_{1}\right) \otimes \cdots \otimes \mathcal{D}_{d}\left(n_{k}\right) \\
\simeq \mid \begin{array}{c}
\simeq I_{k} \otimes I_{n_{1}} \otimes \cdots \otimes I_{n_{k}} \\
I_{n}
\end{array} \\
\qquad \begin{array}{c}
\Omega_{\mathrm{PA}}^{*}\left(\mathcal{F}_{d}(k)\right) \otimes \Omega_{\mathrm{PA}}^{*}\left(\mathcal{F}_{d}\left(n_{1}\right)\right) \otimes \cdots \otimes \Omega_{\mathrm{PA}}^{*}\left(\mathcal{F}_{d}\left(n_{k}\right)\right) \\
\simeq \mid \text { Kunneth }
\end{array} \\
\Omega_{\mathrm{PA}}^{*}\left(\mathcal{F}_{d}(n)\right) \stackrel{\Omega_{\mathrm{PA}}^{*}(\mu)}{\longrightarrow} \Omega_{\mathrm{PA}}^{*}\left(\mathcal{F}_{d}(k) \times \mathcal{F}_{d}\left(n_{1}\right) \times \cdots \times \mathcal{F}_{d}\left(n_{k}\right)\right)
\end{gathered}
$$

where $\mu$ and $\phi$ are the (co)operadic structure maps on $\mathcal{F}_{d}$ and $\mathcal{D}_{d}$ and the vertical maps are quasi-isomorphisms.

On the other hand, $\left\{\bar{I}_{n}: \mathcal{D}_{d}(n) \stackrel{\sim}{\rightarrow} \mathrm{H}^{*}\left(\mathcal{F}_{d}(n) ; \mathbb{R}\right)\right\}_{n \geq 0}$ is a quasi-isomorphism of cooperads. This, combined with Theorem 7.2, is what we mean when we say that the Fulton-MacPherson operad is formal over $\mathbb{R}$. Notice that if we work dually at the level of chains, we can use a monoidal functor to get genuine formality in the category of chain complexes.

In order to prove the formality of the Fulton-MacPherson fanic diagram, we will prove the formality of the morphism $\epsilon_{\#}: \mathcal{F}_{1}^{(0)} \rightarrow \mathcal{F}_{d}$. The key result we need is Lemma 7.3 below. To explain this, let $\mathcal{A S S}=\{\mathbb{R}\}_{n \geq 0}$ be the coassociative cooperad in CDGA. In degree 0 , the vector space $\mathcal{D}_{d}(n)$ is generated by the single admissible graph with $n$ 
external vertices and no edges. These isomorphisms $\mathcal{D}_{d}(n)^{0} \cong \mathbb{R}$ induces a morphism of nonsymmetric cooperads in CDGA

$$
\epsilon: \mathcal{D}_{d} \longrightarrow \mathcal{A S S}
$$

Let $\eta_{n}: \mathbb{R} \rightarrow \Omega_{\mathrm{PA}}^{*}\left(\mathcal{F}_{1}^{(0)}(n)\right)$ be the inclusion of constants in degree 0 .

Lemma 7.3 For $d \geq 3$, diagram

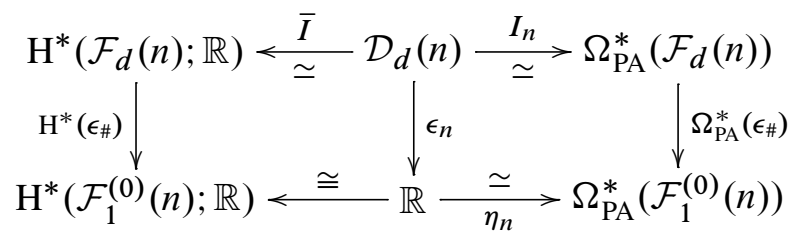

commutes.

Proof The left square is clearly commutative. For the right square in case $n \leq 1$, $\mathcal{D}_{d}(n)$ is concentrated in degree 0 and the diagram clearly commutes. So assuming $n \geq 2$, let $\Gamma \in \mathcal{D}_{d}(n)$ be an admissible graph of positive degree with $n$ external vertices. Assume first that $\Gamma$ has no isolated vertices, ie each external vertex is an endpoint of at least one edge. Let $q$ be the number of internal vertices of $\Gamma$ and recall that every internal vertex has valence at least 3 . Then the number $e$ of edges of $\Gamma$ satisfies

$$
e \geq \frac{1}{2}(n+3 q) \text {. }
$$

Therefore

$$
\begin{aligned}
\operatorname{deg}(I(\Gamma))=\operatorname{deg}(\Gamma) & =e(d-1)-q d \\
& \geq \frac{1}{2}(n+3 q)(d-1)-q d=q\left(\frac{d-3}{2}\right)+n\left(\frac{d-1}{2}\right) .
\end{aligned}
$$

Since $d \geq 3$ this implies $\operatorname{deg}(I(\Gamma)) \geq n$. On the other hand, we have $\operatorname{dim}\left(\mathcal{F}_{1}^{(0)}(n)\right)=$ $n-2$ and so

$$
\operatorname{deg}(I(\Gamma))>\operatorname{dim}\left(\mathcal{F}_{1}^{(0)}(n)\right) .
$$

Since for any semialgebraic set $X, \Omega_{\mathrm{PA}}^{i}(X)=0$ if $i>\operatorname{dim}(X)$, we deduce that

$$
\Omega_{\mathrm{PA}}^{*}(\epsilon)(I(\Gamma))=0 \in \Omega_{\mathrm{PA}}^{*}\left(\mathcal{F}_{1}^{(0)}(n)\right) .
$$

Now suppose that $\Gamma$ has only $j<n$ external vertices which are not isolated. Let $\sigma: \mathcal{D}_{d}(j) \rightarrow \mathcal{D}_{d}(n)$ be the composite of codegeneracies $\sigma_{i}$ inserting the $n-j$ isolated vertices. Hence there exists an admissible graph $\Gamma^{\prime} \in \mathcal{D}_{d}(j)$ with no isolated vertex and such that $\Gamma=\sigma\left(\Gamma^{\prime}\right)$. Consider the map $s: \mathcal{F}_{d}(n) \rightarrow \mathcal{F}_{d}(j)$ obtained as the composition 
of $n-j$ codegeneracies $s^{i}$ that forget the configuration points corresponding to these isolated vertices. Since (co)degeneracies are induced by the operadic structure and $I$ is a map of (almost) cooperads, it is easy to check that the following diagram commutes:

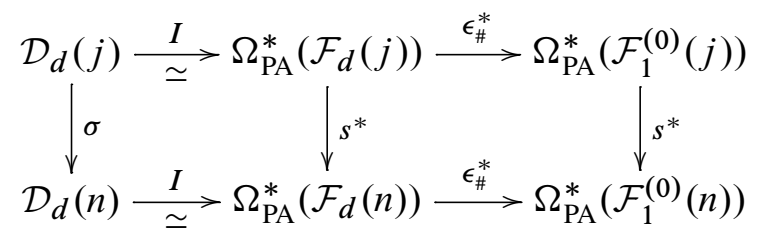

Since $\Gamma^{\prime}$ has no isolated vertices it follows from the previous case that $\epsilon_{\#}^{*}\left(I\left(\Gamma^{\prime}\right)\right)=0$ and so

$$
\epsilon_{\#}^{*}(I(\Gamma))=\epsilon_{\#}^{*}\left(I\left(\sigma\left(\Gamma^{\prime}\right)\right)\right)=s^{*}\left(\epsilon_{\#}^{*}\left(I\left(\Gamma^{\prime}\right)\right)\right)=0 .
$$

We are now ready for the proof of the formality of the Fulton-MacPherson fanic diagram.

Proof of Theorem 7.1 The fanic diagram $\widehat{\mathcal{F}_{d\{n\}}}$ induces a cofanic diagram of CDGAs

$$
\Omega_{\mathrm{PA}}^{*}\left(\widehat{\mathcal{F}}_{d\{n\}}\right): \Phi[n]^{\mathrm{op}} \longrightarrow \text { CDGA. }
$$

We also have a diagram of CDGA cooperads

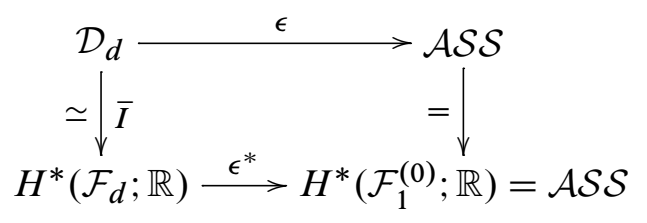

which induces quasi-isomorphic cofanic diagrams

$$
\widehat{\mathcal{D}}_{d\{n\}} \simeq \widehat{H^{*}\left(\mathcal{F}_{d} ; \mathbb{R}\right)_{\{n\}}}: \Phi[n]^{\mathrm{op}} \longrightarrow \mathrm{CDGA}
$$

and of course the cofanic diagram $\widehat{H^{*}\left(\mathcal{F}_{d} ; \mathbb{R}\right)_{\{n\}}}$ is $\mathbb{R}$-formal.

We still need to construct a natural quasi-isomorphism

$$
\widehat{I}: \widehat{\mathcal{D}}_{d\{n\}} \stackrel{\simeq}{\longrightarrow} \Omega_{\mathrm{PA}}^{*}\left(\widehat{\mathcal{F}_{d}}\{n\}\right) .
$$

For a fan $T \in \Phi[n]$ with bead $b$, and for a nonroot vertex $v$ recall that $|v|=$ valence $(v)-1$. Consider the morphism

$$
\begin{aligned}
& I_{|b|} \otimes \otimes_{v \neq b} \eta_{|v|}: \widehat{\mathcal{D}}_{d\{n\}}(T) \cong \mathcal{D}_{d}(|b|) \otimes \otimes_{v \neq b} \mathbb{R} \\
& \longrightarrow \Omega_{\mathrm{PA}}^{*}\left(\mathcal{F}_{d}(|b|)\right) \otimes \otimes_{v \neq b} \Omega_{\mathrm{PA}}^{*}\left(\mathcal{F}_{1}^{(0)}(|v|)\right)
\end{aligned}
$$


and define $\widehat{I}(T)$ as the composite of this map with the Kunneth quasi-isomorphism

$$
\Omega_{\mathrm{PA}}^{*}\left(\mathcal{F}_{d}(|b|)\right) \otimes \otimes_{v \neq b} \Omega_{\mathrm{PA}}^{*}\left(\mathcal{F}_{1}^{(0)}(|v|)\right) \stackrel{\simeq}{\longrightarrow} \Omega_{\mathrm{PA}}^{*}\left(\mathcal{F}_{d}(|b|) \times \prod_{v \neq b} \mathcal{F}_{1}^{(0)}(|v|)\right) .
$$

We need to show that $\hat{I}$ is a natural transformation. Remember from Section 6 the definition of the image of a morphism by the fanic diagram associated to a morphism of (co)operads. Let $e$ be an edge of $T$ emanating from $x$ and ending at $y$, let $i$ be the label of the output $e$ with respect to its origin $x$, and let $\bar{e}$ be the vertex in $\bar{T}=T / E$ corresponding to this contracted edge. Denote by $v$ any vertex of $T$ different from $x$ and $y$ or any vertex of $\bar{T}$ different from $\bar{e}$ (these two sets of vertices are the same). We have three cases: (i) $x=b \neq y$, (ii) $x \neq b=y$, and (iii) $x \neq b \neq y$. We will treat only case (i), the second case being completely analogous and the third simpler. In case (i), $\bar{e}$ is the bead of $\bar{T}$. Consider the diagram

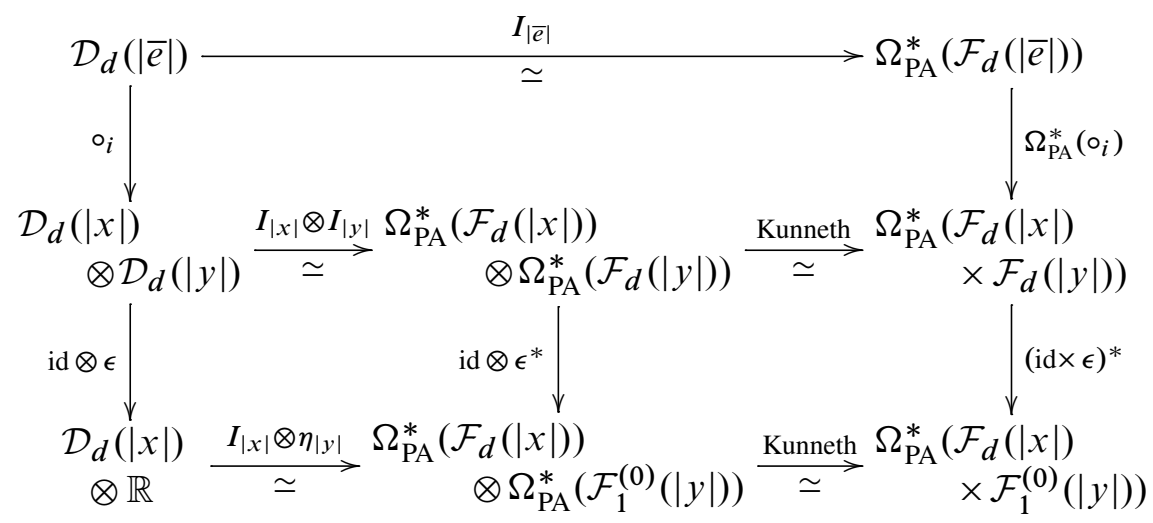

The top rectangle is commutative as a consequence of the commutativity of diagram (7-1) and the definition of the insertion maps $\circ_{i}$ from the (co)operad structures. The left bottom square is commutative by Lemma 7.3, and the right bottom square is commutative by naturality of the Kunneth quasi-isomorphism.

Consider now the outermost square of (7-2) and tensor the left side by $\otimes_{v} \mathbb{R}$ and the right side by $\otimes_{|v|} \Omega_{\mathrm{PA}}^{*}\left(\mathcal{F}_{1}^{(0)}(|v|)\right)$. Applying once more the Kunneth quasi-isomorphism to the right side gives the commutative diagram

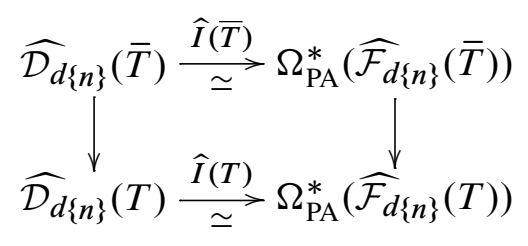

which is what we were after. 
Remark 7.4 Let us explain more precisely what we mean when we say that we cannot prove that the Kontsevich operad is formal as a multiplicative operad and why we need to go through fanic diagrams. Lemma 7.3 proves that the morphism $\epsilon_{\#}: \mathcal{F}_{1}^{(0)} \rightarrow \mathcal{F}_{d}$ is formal, so the weak equivalence (2-5) implies that we have a chain of quasiisomorphisms of morphisms of (almost) cooperads between $A_{\mathrm{PL}}(\mathcal{A S S}) \rightarrow A_{\mathrm{PL}}\left(\mathcal{K}_{d}\right)$ and $\mathrm{H}^{*}(\mathcal{A S S} ; \mathbb{R}) \rightarrow \mathrm{H}^{*}\left(\mathcal{K}_{d} ; \mathbb{R}\right)$. The two cooperads $A_{\mathrm{PL}}\left(\mathcal{K}_{d}\right)$ and $H^{*}\left(\mathcal{K}_{d} ; \mathbb{R}\right)$ are comultiplicative since $A_{\mathrm{PL}}(\mathcal{A S S})=\mathrm{H}^{*}(\mathcal{A S S} ; \mathbb{R})=\{\mathbb{R}\}_{n \geq 0}$. The problem is that, in the chain of quasi-isomorphisms joining $A_{\mathrm{PL}}(\mathcal{A S S})$ and $\mathrm{H}^{*}(\mathcal{A S S} ; \mathbb{R})$, nothing guarantees that all the intermediate CDGAs will be coassociative even if their homologies are. Therefore we cannot apply the dual of the Gerstenhaber-Voronov/McClure-Smith construction to the intermediate CDGAs. One way around this would be to use "simplicial up to homotopy" CDGAs but we wanted to avoid that. Instead, fanic diagrams allow us to work with strictly commutative diagrams.

\section{Proof of the main theorem}

In this section we finally prove Theorem 1.2. We begin with:

Proposition 8.1 For $d \geq 3$ and $n \geq 0$ the rational homology Bousfield-Kan spectral sequence of the cosimplicial replacement $\Pi^{\bullet} \mathcal{K}_{d[n]}$ of the $n$-th truncation of Sinha's cosimplicial space $\mathcal{K}_{d}^{\bullet}$ collapses at the $E^{2}$ page.

Proof By Theorem 7.1, the Fulton-MacPherson fanic diagram $\widehat{\mathcal{F}}_{d\{n\}}$ is $\mathbb{R}$-formal. By diagram (2-5) and Proposition 6.2, we have an equivalence of diagrams $\widehat{\mathcal{F}}_{d\{n\}} \simeq \widehat{\mathcal{K}}_{d\{n\}}$ and by Theorem 6.3 we have $\widehat{\mathcal{K}}_{d\{n\}}=\phi_{n}^{*}\left(\mathcal{K}_{d[n]}\right)$ where $\phi_{n}: \Phi[n] \rightarrow \Delta[n]$ is the left cofinal functor from Theorem 5.8.

Thus the finite diagram $\phi_{n}^{*}\left(\mathcal{K}_{d[n]}\right)$ is $\mathbb{R}$-formal, and so is its cosimplicial replacement by Proposition 3.3. By Proposition 3.2, its homology spectral sequence collapses at the $E^{2}$ page. Since $\phi_{n}$ is left cofinal by Theorem 5.8, we deduce from Proposition 4.5 that the homology spectral sequence of $\Pi^{\bullet} \mathcal{K}_{d[n]}$ collapses at $E^{2}$ page.

We are now ready for the proof of our main theorem. The trick will be to replace $\mathcal{K}_{d}^{\bullet}$ (which we do not know to be formal) by an associated formal cosimplicial space $\Xi^{\bullet}$.

Proof of Theorem 1.2 We first recall some ideas from rational homotopy theory. Let $\mathrm{CDGA}_{1}$ be the full subcategory of CDGA over $\mathbb{Q}$ of simply connected CDGAs of finite type, and let DGL be the category of connected differential graded Lie algebras as in [10, Section 21 (f)]. As explained in [10, Section 22], there are two functors

$$
\mathrm{CDGA}_{1} \stackrel{\mathcal{L}}{\longrightarrow} \mathrm{DGL} \stackrel{C^{*}}{\longrightarrow} \mathrm{CDGA}
$$


where $\mathcal{L}(A)$ is essentially the primitive part of the cobar on the dual coalgebra $\operatorname{hom}(A ; \mathbb{Q})$ and $C^{*}(L)$ is the dual of the bar construction on the enveloping algebra of $L \in \mathrm{DGL}$. The only property of these functors of interest to us is that, for $A \in \mathrm{CDGA}_{1}$, $C^{*}(\mathcal{L}(A))$ is a Sullivan algebra quasi-isomorphic to $A$ [10, Section 22 (e)]. In other words, $C^{*}(\mathcal{L}(-))$ can serve as a cofibrant replacement functor.

We also have a spatial realization functor

$$
|-|: \text { CDGA } \rightarrow \text { Top }
$$

defined in [10, Section 17]. An important property of this functor is that a Sullivan algebra $A$ is naturally weakly equivalent to $A_{\mathrm{PL}}(|A| ; \mathbb{Q})[10$, Section 17 (d)].

Now consider the simplicial CDGA $H^{*}\left(\mathcal{K}_{d}^{\bullet} ; \mathbb{Q}\right)$ and define the cosimplicial space

$$
\Xi^{\bullet}:=\left|C^{*}\left(\mathcal{L}\left(H^{*}\left(\mathcal{K}_{d}^{\bullet} ; \mathbb{Q}\right)\right)\right)\right| .
$$

It is clear from the discussion above that this cosimplicial space is formal and has the same cohomology as $\mathcal{K}_{d}^{\bullet}$.

A more surprising property, arising from the fact that the cohomology algebras of configuration spaces in $\mathbb{R}^{n}$ are Koszul, is that the cosimplicial space $\Xi^{\bullet}$ is also coformal. This is not difficult to prove and details are in [1, Section 4], in particular Corollary 4.3 , noticing that $\Xi^{\bullet}$ is exactly $\left|\chi^{\bullet}\right|$ in that paper. Also explained in that paper is the consequence that there exists an isomorphism of cosimplicial groups

$$
\pi_{*}\left(\Xi^{\bullet}\right) \cong \pi_{*}\left(\mathcal{K}_{d}^{\bullet}\right) \otimes \mathbb{Q} .
$$

We know by Proposition 4.4 that $\mathcal{K}_{d}^{\bullet}$ is well above the diagonal at the $E^{1}$ page and by (8-1) the same is true for $\Xi^{\bullet}$. Therefore the convergence results from Proposition 4.3 hold for both $\mathcal{K}_{d}$ and $\Xi^{\bullet}$.

Since $\Xi^{\bullet}$ is formal, the same is true for $\Pi^{\bullet} \Xi_{[n]}$, so the homology spectral sequence of that cosimplicial replacement collapses at the $E^{2}$ page. But this $E^{2}$ page is clearly isomorphic to the $E^{2}$ page of the homology spectral sequence of $\Pi^{\bullet} \mathcal{K}_{d[n]}$, since the two cosimplicial spaces have the same homology. Moreover, the second spectral sequence also collapses by Proposition 8.1, and both spectral sequences converge by Proposition 4.3. Therefore $\mathrm{H}_{*}\left(\operatorname{hoTot}^{n} \Xi^{\bullet} ; \mathbb{Q}\right) \cong \mathrm{H}_{*}\left(\operatorname{hoTot}^{n} \mathcal{K}_{d}^{\bullet} ; \mathbb{Q}\right)$, and by Proposition 4.3(iii) this implies

$$
\mathrm{H}_{*}\left(\text { hoTot } \Xi^{\bullet} ; \mathbb{Q}\right) \cong \mathrm{H}_{*}\left(\text { hoTot } \mathcal{K}_{d}^{\bullet} ; \mathbb{Q}\right) \text {. }
$$

By formality of $\Xi^{\bullet}$, its rational homology Bousfield-Kan spectral sequence also collapses at the $E^{2}$ page, which is isomorphic to the $E^{2}$ page of $\mathcal{K}_{d}^{\bullet}$. Since both these spectral sequences converge to the isomorphic terms of $(8-2)$, we deduce that the homology spectral sequence of $\mathcal{K}_{d}^{\bullet}$ also collapses at the $E^{2}$ page. 


\section{References}

[1] G Arone, P Lambrechts, V Turchin, I Volić, Coformality and rational homotopy groups of spaces of long knots, Math. Res. Lett. 15 (2008) 1-14 MR2367169

[2] G Arone, P Lambrechts, I Volić, Calculus of functors, operad formality, and rational homology of embedding spaces, Acta Math. 199 (2007) 153-198 MR2358051

[3] S Axelrod, I M Singer, Chern-Simons perturbation theory. II, J. Differential Geom. 39 (1994) 173-213 MR1258919

[4] R Bott, C Taubes, On the self-linking of knots. Topology and physics, J. Math. Phys. 35 (1994) 5247-5287 MR1295465

[5] A K Bousfield, On the homology spectral sequence of a cosimplicial space, Amer. J. Math. 109 (1987) 361-394 MR882428

[6] A K Bousfield, D M Kan, Homotopy limits, completions and localizations, Lecture Notes in Math. 304, Springer, Berlin (1972) MR0365573

[7] A S Cattaneo, P Cotta-Ramusino, R Longoni, Configuration spaces and Vassiliev classes in any dimension, Algebr. Geom. Topol. 2 (2002) 949-1000 MR1936977

[8] F R Cohen, T J Lada, J P May, The homology of iterated loop spaces, Lecture Notes in Math. 533, Springer, Berlin (1976) MR0436146

[9] P Deligne, P Griffiths, J Morgan, D Sullivan, Real homotopy theory of Kähler manifolds, Invent. Math. 29 (1975) 245-274 MR0382702

[10] Y Félix, S Halperin, J-C Thomas, Rational homotopy theory, Graduate Texts in Math. 205, Springer, New York (2001) MR1802847

[11] G Gaiffi, Models for real subspace arrangements and stratified manifolds, Int. Math. Res. Not. (2003) 627-656 MR1951400

[12] M Gerstenhaber, A A Voronov, Homotopy G-algebras and moduli space operad, Internat. Math. Res. Notices (1995) 141-153 MR1321701

[13] E Getzler, J D S Jones, Operads, homotopy algebra and iterated integrals for double loop spaces arXiv:hep-th/9403055

[14] PG Goerss, J F Jardine, Simplicial homotopy theory, Progress in Math. 174, Birkhäuser Verlag, Basel (1999) MR1711612

[15] T G Goodwillie, J R Klein, MS Weiss, Spaces of smooth embeddings, disjunction and surgery, from: "Surveys on surgery theory, Vol. 2", (S Cappell, A Ranicki, J Rosenberg, editors), Ann. of Math. Stud. 149, Princeton Univ. Press (2001) 221-284 MR1818775

[16] M Kontsevich, Vassiliev's knot invariants, from: "I M Gel'fand Seminar", (S Gel'fand, S Gindikin, editors), Adv. Soviet Math. 16, Amer. Math. Soc. (1993) 137-150 MR1237836 
[17] M Kontsevich, Feynman diagrams and low-dimensional topology, from: "First European Congress of Mathematics, Vol. II (Paris, 1992)”, Progr. Math. 120, Birkhäuser, Basel (1994) 97-121 MR1341841

[18] M Kontsevich, Operads and motives in deformation quantization, Lett. Math. Phys. 48 (1999) 35-72 MR1718044 Moshé Flato (1937-1998)

[19] M Kontsevich, Y Soibelman, Deformations of algebras over operads and the Deligne conjecture, from: "Conférence Moshé Flato 1999, Vol. I (Dijon)", (G Dito, D Sternheimer, editors), Math. Phys. Stud. 21, Kluwer Acad. Publ., Dordrecht (2000) 255-307 MR1805894

[20] P Lambrechts, V Turchin, Homotopy graph-complex for configuration and knot spaces, Trans. Amer. Math. Soc. 361 (2009) 207-222 MR2439404

[21] P Lambrechts, V Turchin, I Volić, Associahedron, cyclohedron and permutohedron as compactifications of configuration spaces, Bull. Belg. Math. Soc. Simon Stevin 17 (2010) 303-332 MR2663475

[22] P Lambrechts, I Volić, Formality of the little $N$-discs operad, Preprint (2010) Available at http://palmer.wellesley.edu/ ivolic/pages/papers.html

[23] M Markl, A compactification of the real configuration space as an operadic completion, J. Algebra 215 (1999) 185-204 MR1684178

[24] M Markl, S Shnider, J Stasheff, Operads in algebra, topology and physics, Math. Surveys and Monogr. 96, Amer. Math. Soc. (2002) MR1898414

[25] J P May, The geometry of iterated loop spaces, Lectures Notes in Math. 271, Springer, Berlin (1972) MR0420610

[26] J E McClure, J H Smith, A solution of Deligne's Hochschild cohomology conjecture, from: "Recent progress in homotopy theory (Baltimore, MD, 2000)", (D M Davis, J Morava, G Nishida, W S Wilson, N Yagita, editors), Contemp. Math. 293, Amer. Math. Soc. (2002) 153-193 MR1890736

[27] P Salvatore, Configuration operads, minimal models and rational curves, $\mathrm{PhD}$ thesis, Oxford University (1998)

[28] K P Scannell, D P Sinha, A one-dimensional embedding complex, J. Pure Appl. Algebra 170 (2002) 93-107 MR1896343

[29] D P Sinha, Manifold-theoretic compactifications of configuration spaces, Selecta Math. (N.S.) 10 (2004) 391-428 MR2099074

[30] D P Sinha, Operads and knot spaces, J. Amer. Math. Soc. 19 (2006) 461-486 MR2188133

[31] D P Sinha, The topology of spaces of knots: cosimplicial models, Amer. J. Math. 131 (2009) 945-980 MR2543919

[32] S Smale, The classification of immersions of spheres in Euclidean spaces, Ann. of Math. (2) 69 (1959) 327-344 MR0105117 
[33] V Tourtchine (Turchin), On the homology of the spaces of long knots, from: "Advances in topological quantum field theory”, (J M Bryden, editor), NATO Sci. Ser. II Math. Phys. Chem. 179, Kluwer Acad. Publ., Dordrecht (2004) 23-52 MR2147415

[34] V Tourtchine (Turchin), What is one-term relation for higher homology of long knots, Mosc. Math. J. 6 (2006) 169-194, 223 MR2265954

[35] V Tourtchine (Turchin), On the other side of the bialgebra of chord diagrams, J. Knot Theory Ramifications 16 (2007) 575-629 MR2333307

[36] V A Vassiliev, Cohomology of knot spaces, from: "Theory of singularities and its applications", (V I Arnol'd, editor), Adv. Soviet Math. 1, Amer. Math. Soc. (1990) 23-69 MR1089670

[37] V A Vassiliev, Invariants of knots and complements of discriminants, from: "Developments in mathematics: the Moscow school", (V I Arnol'd, M Monastyrsky, editors), Chapman \& Hall, London (1993) 194-250 MR1264426

[38] V A Vassiliev, Homology of $i$-connected graphs and invariants of knots, plane arrangements, etc, from: “The Arnoldfest (Toronto, ON, 1997)", (E Bierstone, B Khesin, A Khovanskii, J E Marsden, editors), Fields Inst. Commun. 24, Amer. Math. Soc. (1999) 451-469 MR1733588

[39] C A Weibel, An introduction to homological algebra, Cambridge Studies in Advanced Math. 38, Cambridge Univ. Press (1994) MR1269324

Université Catholique de Louvain

2 Chemin du Cyclotron, B-1348 Louvain-la-Neuve, Belgium

Department of Mathematics, Kansas State University

Manhattan KS 66506, USA

Department of Mathematics, Wellesley College

106 Central St, Wellesley MA 02481, USA

lambrechts@math.ucl.ac.be, turchin@ksu.edu, ivolic@wellesley.edu

http://milnor.math.ucl.ac.be/plwiki,

http://www.math.ksu.edu/ turchin/, http://palmer.wellesley.edu/ ivolic

Proposed: Haynes Miller

Seconded: Ralph Cohen, Bill Dwyer
Received: 26 November 2009

Accepted: 11 August 2010 